\title{
Rapid and transient evolution of local adaptation to seasonal host fruits in an invasive fly
}

\author{
Short title: Rapid local adaptation in Drosophila suzukii
}

Laure Olazcuaga $^{1,2}$, Julien Foucaud ${ }^{1}$, Candice Deschamps ${ }^{1}$, Anne Loiseau ${ }^{1}$, Jean-Loup Claret $^{1}$, Romain Vedovato ${ }^{1}$, Robin Guilhot ${ }^{1}$, Cyril Sevely ${ }^{3}$, Mathieu Gautier ${ }^{1}$, Ruth A. Hufbauer ${ }^{1,2}$, Nicolas O. Rode ${ }^{1,4}$ and Arnaud Estoup ${ }^{1,4}$

${ }^{1}$ CBGP, Univ Montpellier, CIRAD, INRAE, Institut Agro, IRD, Montpellier, France

${ }^{2}$ Department of Agricultural Biology, Colorado State University, Fort Collins, Colorado 80523, USA

${ }^{3}$ Chambre d'agriculture de l'Hérault, Lattes, France

${ }^{4}$ These authors contributed equally to this work

Corresponding authors: Laure Olazcuaga, Nicolas O. Rode and Arnaud Estoup E-mail: olaz.laure@gmail.com, nicolas.rode@inrae.fr, arnaud.estoup@inrae.fr

\section{Corresponding author: Laure Olazcuaga}

CBGP - 755 avenue du Campus Agropolis

CS 30016 - 34988 Montferrier sur Lez cedex

France

Phone: +33499623346

E-mail: olaz.laure@gmail.com

\section{Keywords}

Adaptive phenotypic plasticity; Drosophila suzukii; genetic effects; host fruits; local adaptation; reciprocal common garden experiment 


\section{Abstract}

Both adaptive phenotypic plasticity and local adaptation can influence the match between phenotypic traits and local environmental conditions. Theory predicts that coarse-grained environments, which are stable for multiple generations, promote local adaptation, while finegrained environments, in which individuals encounter more than one environment in their lifetime, favor adaptive phenotypic plasticity. When the heterogeneity of the environment is spatially and/or temporarily intermediate, with periods of environmental stability from one to only a few generations, the relative contributions of local adaptation and adaptive phenotypic plasticity in enabling individuals' phenotypes to match the environments they encounter remains unclear. Here, we used Drosophila suzukii as a model system to evaluate the relative influence of genetic and plastic effects on this match in heterogeneous environments with an intermediate grain. This pest insect can develop within different fruits, and persists throughout the year in a given location on a succession of different host fruits, each one being available for only a few generations. Using reciprocal common environment experiments of natural D. suzukii populations collected from cherry, strawberry and blackberry, we found that both oviposition preference and offspring performance were higher on medium made with the fruit from which the population originated, than on media made with alternative fruits. This pattern remained after two generations in the laboratory, suggesting that genetic effects predominate over plastic effects. Our study demonstrates that spatially and temporally variable selection does not prevent the rapid evolution of local adaptation in natural populations. The speed and strength of adaptation may be facilitated by several mechanisms including a large effective population size and strong selective pressures imposed by host plants. 


\section{Impact Summary}

Natural populations often exhibit good "fit" to the environment they are in. However, environments change over both time and space, and following change, the fit between a population and its environment may be poor. A question of long-standing interest to evolutionary biologists is, how do populations track changing environments to maintain fitness? Two main mechanisms are known: $(i)$ plastic shifts, or adaptive phenotypic plasticity, in which traits immediately change in response to environmental change, and (ii) genetic shifts in the form of local adaptation, in which traits change over time through differences in fitness of individuals harboring different genetic variants. Plasticity is common when environments change over the course of an individual lifetime, while adaptation is common when environments change over the course of multiple generations. However, many environments change at an intermediate pace, and it is unclear whether plasticity or adaptation are more vital to maintaining fitness under such conditions.

Drosophila suzukii is well-suited to evaluating the relative importance of plasticity and adaptation in response to an intermediate pace of environmental change. This species experiences an environment that shifts every 1-4 generations as host fruits change over time and space. Here, we studied natural populations of $D$. suzukii collected from different hosts. Using reciprocal common environment experiments, we evaluated their fitness on their source and alternative hosts.

Drosophila suzukii populations were most fit on their source host, successfully tracking an intermediate pace of environmental change. We developed a new statistical method to quantify the contributions of adaptive plasticity and local adaptation in determining fitness. We found that fitness was maintained via local adaptation to each new host in succession. This study provides a novel statistical tool that can be applied to other systems, 
bioRxiv preprint doi: https://doi.org/10.1101/2022.03.01.482503; this version posted March 4, 2022. The copyright holder for this preprint (which was not certified by peer review) is the author/funder, who has granted bioRxiv a license to display the preprint in perpetuity. It is made available under aCC-BY-NC-ND 4.0 International license.

and highlights that spatially and temporally variable selection does not prevent local adaptation and, on the contrary, illustrates how rapid the adaptive process can be. 


\section{Introduction}

Adaptation is the evolutionary process whereby organisms come to match environmental conditions in ways that enhance fitness. When selection differs between environments, adaptation to the home environment may result in reduced fitness in other environments (e.g., Via, 1991; Torres Dowdall et al., 2012). Such a pattern of higher fitness in the natal environment than in alternate environments is typically understood to be due to local adaptation of genetically differentiated populations. However some researchers (Torres Dowdall et al., 2012; Yampolsky et al., 2014; Rago et al., 2019; Bonnet et al., 2021; Enbody et al., 2021) have found that this pattern can arise through adaptive phenotypic plasticity. Adaptive phenotypic plasticity allows the tracking of environmental change within one or a couple of generations without genetic change (i.e., through behavioral or developmental plasticity, maternal or paternal effects, or trans-generational plasticity; Price et al., 2003). In contrast, local adaptation results in genetic differentiation among populations specialized for different environments (Kawecki \& Ebert, 2004).

Theory suggests that environmental grain largely determines the relative importance of adaptive phenotypic plasticity (plastic effects) and local adaptation (genetic effects) in matching the phenotype to its environment (hereafter "phenotypic adaptation") (Levins, 1968). Environmental grain indicates the speed at which the environment experienced by an individual changes, due to alteration of the environment itself or to the dispersal of the individual across spatially varying environments (Gillespie, 1974). Hence, the environmental grain depends upon the magnitude and frequency of environmental changes, and on the organism's life history traits (i.e., generation time, dispersal rate and distance; (Levins, 1968; Botero et al., 2015; Lande, 2015; Tufto, 2015; Scheiner, 2016; Edelaar et al., 2017). Differences in environmental grain are often categorized as fine or coarse. A fine-grained environment refers to situations where individuals encounter more than one environmental 
condition during their development or throughout their lifetime (e.g., when dispersal rates are higher than the levels of spatial heterogeneity). In contrast, a coarse-grain environment refers to situations where the environment remains constant throughout an individual's lifetime and for several consecutive generations (i.e., the generation time is shorter than the temporal scale of environmental fluctuations).

In fine-grained environments, adaptive phenotypic plasticity is favored as it modifies phenotypes more rapidly than selection on standing genetic variation (Levins, 1968;

Gillespie, 1974; Botero et al., 2015; Tufto, 2015). Adaptive phenotypic plasticity is expected to evolve when environmental conditions can be assessed through reliable cues (i.e., accurate environmental information about future selection on the expressed plastic phenotype; (Gavrilets \& Scheiner, 1993; Jong, 1999; Tufto, 2000), and when the benefits of plasticity outweigh its costs (Dewitt et al., 1998; Pigliucci, 2001; DeWitt \& Scheiner, 2004). Indeed, maintaining plasticity may require energy and material expenses (e.g., cost of maintaining a sensory and regulatory machinery; Dewitt et al., 1998; Van Buskirk \& Steiner, 2009; Auld et al., 2010).

In coarse-grained environments, local adaptation and genetic differentiation are expected to evolve as they do not require reliable environmental cues and might be less costly than plastic responses (Dewitt et al., 1998; Auld et al., 2010). However, the ability to respond to selection and the magnitude of local adaptation depends on genetic variation within populations as well as gene flow among populations, and the environmental and phenotypic divergence among populations (Lande, 1976; Endler, 1977; Slatkin, 1985; García-Ramos \& Kirkpatrick, 1997).

Fine- and coarse-grained environments are ends of a continuum, and many organisms likely experience an intermediate environmental grain. Phenotypic responses to intermediategrained environments - with periods of environmental stability from one to only a few 
generations - should be shaped by a combination of genetic and plastic responses (Schmid, 1992). Moreover, the relative contribution of genetic and plastic responses is expected to change through time, as both the speed of their response to selection and their relative costbenefits are likely to differ. Adaptive phenotypic plasticity may at first allow populations to establish and persist in a novel environment (Bradshaw, 1965; Via \& Lande, 1985; Gotthard \& Nylin, 1995; Robinson \& Dukas, 1999; Price et al., 2003; Ghalambor et al., 2007; Uller, 2008; Chevin et al., 2010; Sun et al., 2020), before adaptive genetic differentiation evolves. If plasticity is costly, its magnitude is expected to decrease over time so at a given point in the process of local adaptation, locally adapted phenotypes are only determined and maintained by genetic effects (a process referred to as "genetic assimilation"; (Waddington, 1953; Pigliucci et al., 2006; Lande, 2009).

Adaptive phenotypic plasticity can only be distinguished from local adaptation by performing reciprocal common environment experiments over multiple generations, by measuring performance of populations in their original environment (sympatry) as well as in other environments (allopatry), and this over several generations ( Fig. 1A vs 1B). The relative importance of genetic and plastic responses in matching the phenotype of natural populations to their environment is beginning to be studied in fine- and coarse-grained environments (Rago et al., 2019; Bonnet et al., 2021; Enbody et al., 2021), and to our knowledge, no study has investigated the importance of local adaptation and phenotypic plasticity when populations evolve in an intermediate-grained environment. Three main experimental and statistical reasons account for this lack of empirical evidence. First, selective pressures are hard to control in natura, as they can vary both spatially and temporally (Rausher, 1988; Fry, 1996; Hansen et al., 2006; Barghi et al., 2020). This issue is best tackled by performing reciprocal common environment experiments in the laboratory or other controlled environment where local adaptation to a given biotic or abiotic factor of 
interest can be tested on populations from different geographical locations (Fig. 1A;

(Turesson, 1922; Kawecki \& Ebert, 2004; Hereford, 2009). Second, local adaptation can be masked by variation in performance among populations due to environmental effects (nonadaptive phenotypic plasticity; Fig. 1C). Indeed, local adaptation can only be distinguished from non-adaptative and adaptive phenotypic plasticity by performing reciprocal common environment experiments. In these experiments, individuals from populations from different geographical locations are raised in the same environment over one or several generations (Kawecki \& Ebert, 2004). Third, although multi-generation laboratory experiments allow estimating both genetic and plastic responses (Merila \& Hendry, 2014), reliable statistical tools to estimate and test their relative contribution to the performance of populations in various environments are currently lacking.

Phytophagous insects are valuable biological models to investigate the relative contribution of genetic and plastic responses to varying natural environmental conditions. The host plants of these insects represent heterogeneous resources that vary spatially and temporally in both availability and quality. Oviposition preference for and offspring performance on a host plant are important fitness components that shape host use and specialization in phytophagous insects (Jaenike, 1978; Ravigné et al., 2009) and can either be plastic (e.g., learned preference for the habitat encountered as offspring; Dury \& Wade, 2019) or genetic (e.g., preference of individuals for the environment they are the best adapted to; Ravigné et al., 2009). In the present study, we focused on the spotted wing drosophila, Drosophila suzukii, a generalist crop pest with a broad range of host fruits (Lee et al., 2011). This species persists throughout the growing season in a given location on a succession of different host species as they become available (Kenis et al., 2016). Hence, wild populations of D. suzukii evolve in an environment of intermediate grain in which they spend three or four generations on a given host fruit (estimate based on fruit seasonalities and on D. suzukii's 
development time on each fruit; Burrack et al., 2013; Poyet et al., 2015; Aly, 2018). In contrast to most other generalist Drosophila species, the hosts used by D. suzukii are well known (Lee et al., 2011; Walsh et al., 2011). Hence, wild D. suzukii meta-populations represent tractable systems to experimentally estimate the relative contributions of genetic and plastic effects to phenotypic adaptation.

To characterize the phenotypic adaptation in this species, we sampled natural populations on different fruits to perform a reciprocal common environment experiment over multiple generations in the laboratory. We tested for a pattern of higher performance on or oviposition preference for the source fruit (hereafter sympatric) than on other fruits (hereafter allopatric). To do this, we developed a new statistical method allowing estimation of the relative contributions of plastic and genetic effects in this phenotypic adaptation. Additionally we examined if oviposition preference and offspring performance traits are correlated, which could speed the evolution of their co-evolution (Jaenike, 1978).

\section{Material and methods}

\section{Population sampling and laboratory maintenance}

We investigated the adaptation of natural D. suzukii populations to three host plants: Prunus avium (cherry), Fragaria $\times$ ananassa Duch (strawberry) and Rubus fruticosus (blackberry). We chose these fruit species to represent agronomically important crops (cherry, strawberry) and a wild host fruit considered to be an important reservoir (blackberry; Lee et al., 2011; Poyet et al., 2015). In the Northern hemisphere, most varieties of cherry and strawberry are available mainly from the end of the spring to the end of the summer, while blackberry is mostly available from the middle of the summer to the middle of the fall. These three species cover the main active period of $D$. suzukii in the Northern hemisphere (i.e., from May to

October; Walsh et al., 2011). 
Between May 2018 and October 2018, we collected fruits from a total of 47 sites in the South of France (Fig. 2, Table S6). The sampled sites included 20, 12, and 15 sites for cherry, strawberry, and blackberry, respectively. For each host plant, fruits were collected towards the end of the production season of the plant to maximize the number of generations over which flies could adapt to each fruit. Field-collected fruits of each population were brought back to the laboratory and kept at $21^{\circ} \mathrm{C}, 65 \%$ relative humidity and a $18: 6$ (L:D) hour cycle in large plastic cages (volume $~ 90 \mathrm{~L}$ ) until adult D. suzukii flies emerged. This sampling scheme was used consistently throughout the entire experiment. Only the sites from which more than 150 individuals emerged were included in the experiments, leaving a total of 25 sites from the original 47 ( 9 cherry, 3 strawberry, and 13 blackberry sites).

\section{Reciprocal common environment experiment}

We measured female preference and offspring performance of field-collected flies and lab reared flies on purees made from sympatric and allopatric fruit (Fig. 3).

To maintain a constant quality of all fruits throughout the experiment (experimental measurements took place over several months), we prepared media using frozen purees of each of the three fruits rather than whole fruits. $600 \mathrm{ml}$ of each fruit puree was supplemented with $400 \mathrm{ml}$ of sterile deionized water, $1 \%(\mathrm{wt} / \mathrm{vol})$ of agar, $6 \%$ of inactive malted brewer's yeast, and $1.5 \%$ of yeast extract. We then added an antimicrobial solution, which consisted of $6 \mathrm{ml}$ of $99 \%$ proprionic acid, $10 \mathrm{ml}$ of $96 \%$ ethanol and $0.1 \%$ of methylparaben sodium salt. For the common environment, we used a standard laboratory fly food (i.e., "German food"; Backhaus et al. 1984) including $1000 \mathrm{ml}$ of sterile deionized water, 1\% (wt/vol) of agar, 6\% of dextrose, $3 \%$ sucrose, $8 \%$ of inactive malted brewer's yeast, $2 \%$ of yeast extract, $2 \%$ of peptone, $0.05 \%$ of $\mathrm{MgSO}_{4}$, and $0.05 \%$ of $\mathrm{CaCl}_{2}$, and $16 \mathrm{ml}$ antimicrobial solution $(6 \mathrm{ml}$ of $99 \%$ proprionic acid, $10 \mathrm{ml} 96 \%$ of ethanol and $0.1 \%$ of methylparaben sodium salt). See Olazcuaga et al., 2019 for product references. 
For each site, we collected adult flies after the peak of emergence from the fieldcollected fruits (usually within four days after the first adults emerged). We kept only $D$. suzukii individuals and discarded other Drosophila species (Hauser, 2011). We placed adults (hereafter referred to as "G0") in cages with organic commercial fruits corresponding to the fruits from which the population originated and allowed adults to mature for seven days (the absence of prior $D$. suzukii infestation of these fruits was assessed by rigorous visual scrutiny). We performed reciprocal common environment experiments in the laboratory using (i) seven-day-old (+/- two days) G0 adults that emerged from field-collected fruits and (ii) seven-day-old (+/- one day) G2 adults obtained after two generations of maintenance in a common environment (i.e., "German food”). For each population, we estimated female preference and offspring performance in artificial fruit media composed of either the fruit from which the population was sampled (sympatric treatment) or a different fruit (allopatric treatment). We measured oviposition preference and oviposition stimulation (see below) in G0 and G2, as proxies for female preference and we measured egg-to-adult survival in G1 and G3, as a proxy for offspring performance.

Oviposition preference was measured as the number of eggs laid on different fruits by females in an arena that contained 12 fruit media. Adult flies were placed in these arenas in groups of 20, and allowed 24 hours for oviposition, as in Olazcuaga et al. (2019, 2021). We used a no-choice assay to measure the oviposition response of females to individual fruits (hereafter oviposition stimulation), as the number of eggs laid when placing 20 adults for 24 hours in a vial with one of the three fruits. Eggs were counted under dissecting microscopes. For G0, we used on average 7.8 arenas per population to estimate oviposition preference and an average of 7.5 tubes per population for the other traits. For G2, we used 7.9 arenas and 8.0 tubes, respectively. 
Offspring performance was measured on each fruit as egg-to-adult survival. We quantified egg-to-adult survival as the proportion of eggs in the no-choice assay that resulted in the emergence of adults 16 days after oviposition (more than $0.95 \%$ of individuals emerge within 16 days on cherry, strawberry, or blackberry media; Fig. 4 in Olazcuaga et al., 2019).

Our field sampling design followed the seasonality of each fruit. As a result, reciprocal common environment experiments of each population were performed at different dates. To test for potential temporal variability in experimental conditions, we used an inbred D. suzukii inbred line (WT3; Chiu et al., 2013) as a control. Together with each wild population, we measured oviposition preference, oviposition stimulation and offspring performance in this control population. Analyses of each of the three traits of interest showed that temporal variation was of the same order of magnitude as the variation within a given date (Appendix S1).

\section{Statistical methods}

The analyses of oviposition preference and oviposition stimulation (log-transformed number of eggs; Miller, 1997) and offspring performance (arc-sinus-transformed egg-to-adult survival; McDonald, 2014) were performed using the R statistical software (R Core Team, 2014). For offspring performance, 51 tubes had more adults than the number of eggs counted ( 50 of 541 tubes for G0 and 1 of 602 for G2), and were hence removed from analyses of the emergence rate. Analyses that assumed an emergence rate of 1 for these 51 observations provided the same qualitative results.

\section{Testing for trait differences between sympatric and allopatric fruit media}

For each trait and each generation separately, we tested whether populations had a higher preference for or performance in sympatric than in allopatric environments. First, for each population, we plotted the average trait in allopatric environments against the same average trait in sympatric environments. To control for potential confounding effects, we used the 
residuals from a linear model that accounted for variation in quality among fruit media (and arena identity for oviposition preference, or number of eggs for offspring performance). To further examine variation in quality among populations, we also performed a Principal Component Analysis for each trait and each generation separately. Second, we used the $F$-test method of Blanquart et al. (2013) to test for differences in oviposition preference, oviposition stimulation or offspring performance of populations between sympatric and allopatric environments. Briefly, this method consists of fitting an interaction between all pairs of populations and environments on a fitness-related trait and testing whether a factor that contrasts sympatric $v s$. allopatric combinations ( $S A$ effect) explains a significant portion of the variance of this interaction; see Appendix S2 for details. This method thus indicates when there are differences in traits between sympatric and allopatric environments. Positive estimates of the $S A$ effect indicate local adaptation, while negative estimates indicate a pattern of local maladaptation.

\section{A new method to detect and quantify adaptive phenotypic plasticity and local adaptation}

\section{simultaneously}

To evaluate the relative contributions to preference and performance of adaptive phenotypic plasticity and local adaptation, it was necessary to analyze the data across the G0/G1 and G2/G3 generations. When measured on adults that emerged from field-collected fruits (G0/G1), phenotypic differences among populations can be driven both by plastic responses to the natural environment (potentially including adaptive phenotypic plasticity) and by genetic differences among populations (potentially including local adaptation). When measured on adults after two generations of common environment (G2/G3), phenotypic differences among populations are likely to have a genetic basis, as potential maternal and grand-maternal environmental effects are standardized across populations. Although transgenerational plasticity and other non-genetic factors such as vertically transmitted 
symbionts can still be present, we use the term "genetic effects" hereafter for the sake of clarity. To evaluate the relative contributions to preference and performance of adaptive phenotypic plasticity and local adaptation, we developed new approaches to visualize and test for genetic and plastic effects.

To visually illustrate genetic and plastic effects in preference, we estimated the mean of genetic effects based on preference data from $\mathrm{G} 2$ and the mean of plastic effects as the difference in preference between G0 and G2, after controlling for potential confounding variables using a linear model as described above (see Appendix S3 for details). Similarly, for performance, we estimated the mean genetic effects from $\mathrm{G} 3$, and the mean of plastic effects as the difference between G1 and G3.

To statistically test for genetic and plastic effects, we modified the $F$-test of Blanquart et al. (2013) to test whether oviposition preference, stimulation and offspring performance of populations was on average different in sympatric and in allopatric environments and whether this effect was either genetic and present in both generations or was plastic and present in the first generation (G0 or G1), but not the second generation (G2 or G3). To this end, we derived two different $F$-tests for genetic and plastic effects respectively ( $F_{\text {genetic }}$ and $F_{\text {plastic }}$; see Appendix S4 for details). To test the general use of this method, we performed computer simulations, which showed that both plastic and genetic effects can be reliably detected using our method under both balanced and unbalanced experimental designs, with a false positive rate for the detection of plastic and genetic effects <3\% (Fig. S5; Tables S3, S4 and S5).

For oviposition preference, we fitted the following ANOVA model on the log-number of eggs, $y_{i j k l m:}$

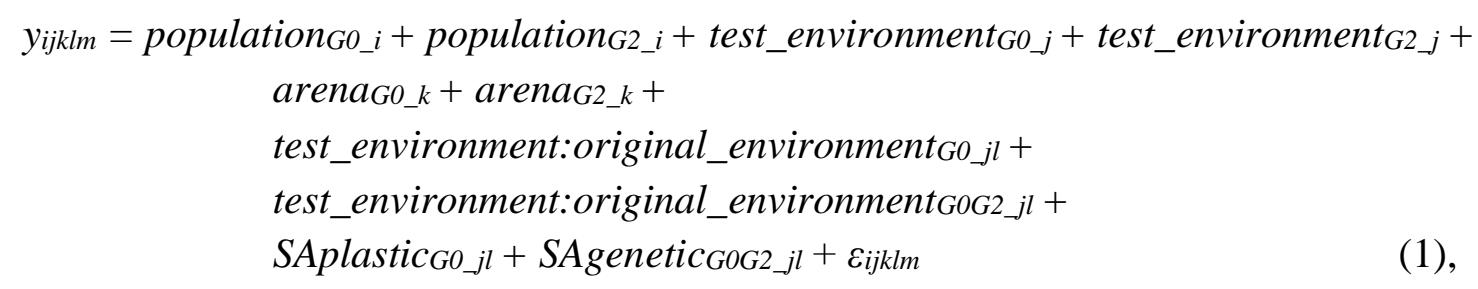


where fixed effects included the effect of the $i$ th population in either G0 or G2 (population $0_{-} i$ or population ${ }_{G \_} i$ with $i=1, \ldots, 25$ ), the effect of the $j$ th environment (test_environment ${ }_{G} \_j$ or test_environment ${ }_{G 2} j$ with $j=1, \ldots, 3$ for blackberry, cherry and strawberry, respectively) and the effect of the $k$ th arena in either G0 or G2 (arenaG0_k or $_{\text {arena }} \_k$ with $k=1, \ldots, 179$ in G0 and $k=1, \ldots, 190$ in G2) to account for differences in quality among populations, among fruit media and among arenas for each generation separately (G0 and G2 for preference traits, and G1 and G3 for offspring performance). Fixed effect also included the interaction between the $j$ th test environment and the $l$ th original environment that is only observed in G0 (test_environment:original_environment ${ }_{G} \_j l$, with $l=1, \ldots, 3$ for populations originating from blackberry, cherry and strawberry, respectively), the interaction between the $j$ th test environment and the $l$ th original environment that is only observed in both G0 and G2 (test_environment:original_environment ${ }_{G 0 G 2 \_j l}$ ), a sympatric vs. allopatric effect that measures adaptive phenotypic plasticity in G0 (SAplastic $\left.G_{-} j l\right)$, a sympatric $v s$. allopatric effect that measures local adaptation in both G0 and G2 (SAgeneticG0G2_jl), and a random error to account for the variation among observations from the same arena ( $\varepsilon_{i j k l m}$, normally distributed with a mean of zero and variance $\sigma^{2}$ res). As with Blanquart's method, this new method allows to detect if there is a significant difference between allopatric and sympatric environments. A significant difference with a positive estimate of SAplastic 60 and SAgeneticG0G2 allows to show adaptive phenotypic plasticity and local adaptation respectively. We either analyzed the entire dataset (12 fruits) or focused on a smaller dataset with the three fruits of interest (cherry, strawberry, blackberry). Results were similar in both analyses so we only present the results with only the three fruits of interest.

For oviposition stimulation, we fitted the same model without the $k$ th arena effect. For offspring performance, we fitted the same model with G1 and G3. We replaced the $k$ th arena 
effect by the log-transformed initial number of eggs (eggsk) to account for the potential effect of egg density on offspring performance.

To estimate the level of adaptive phenotypic plasticity, we designed a new metric based on the proportion of variance of the interaction test_environment:original_environment ${ }_{G 0} j l$ explained by SAplasticG0_jl:

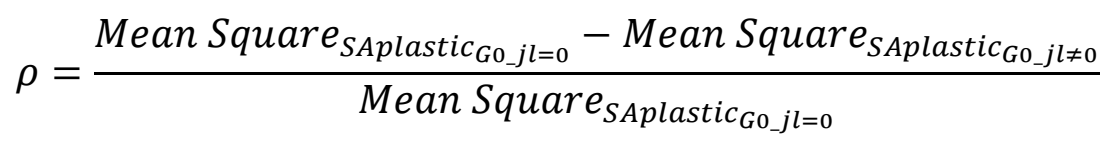

Similarly, the level of local adaptation was estimated based on the proportion of variance of the interaction test_environment:original_environmentG0G2_jl explained by SAgeneticG0G2_jl:

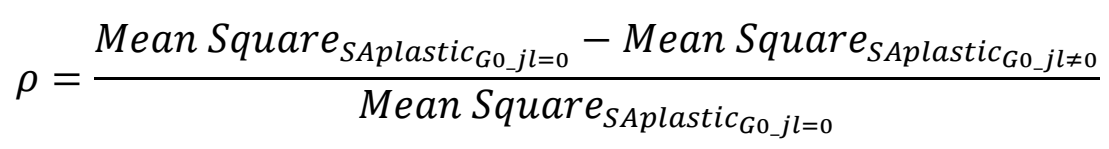

As we did not detect any significant adaptive phenotypic plasticity (see results), we retrospectively performed a simulation-based analysis to estimate our power to detect the presence of adaptive phenotypic plasticity with our experimental design (Appendix S5).

\section{$\underline{\text { Testing for correlations }}$}

We tested for two sets of correlations. First, we evaluated whether there is a correlation in oviposition preference, oviposition stimulation or offspring performance of populations across generations (Appendix S6). Second, to examine whether the evolution of preference and performance traits are independent or rather if they coevolve together, we tested for a correlation between oviposition preference and offspring performance between G0 and G1, and between G2 and G3 (Appendix S7). 


\section{Results}

\section{Test for trait differences between sympatric and allopatric fruit media}

Across the three traits of interest (oviposition preference, oviposition stimulation and offspring performance) and for each generation separately, we found that populations with the highest trait value in sympatry also had the highest trait value in allopatry (Fig. 4; Fig. S3). This correlation of trait values across fruits indicates a strong variation in intrinsic quality among populations and underlines the importance of statistically accounting for this variation when testing for adaptive phenotypic plasticity and local adaptation (Appendix S2). This variation among populations was higher in $\mathrm{G} 0$ than in $\mathrm{G} 2$ for oviposition stimulation (Fig. 4C vs. 4D) and higher in G1 than in G3 for offspring performance (Fig. 4E vs. 4F), suggesting the presence of phenotypic plasticity in G0 and G1. In contrast, variation among populations was not higher in G0 than in G2 for oviposition preference (Fig. 4A vs. 4B).

In G0 and G1, the values of the three traits of interest did not significantly differ between sympatric and allopatric fruit media (Fig. 4A,C,E, Fig. S4A,C,E). In G2 and G3, most of the populations preferred to oviposit and had higher offspring performance in sympatric than in allopatric fruit media (i.e., most populations are under the dashed bisector line in Fig. 4B, F). This pattern was particularly strongly significant for offspring performance $\left(F_{1,3}=87.99, P=0.0026\right.$; Fig. $\left.\mathrm{S} 4 \mathrm{~F}\right)$, but not as strongly significant for oviposition preference $\left(F_{1,3}=11.55, P=0.043\right.$; Fig. S4B). In contrast, the oviposition stimulation of most populations was not stronger on sympatric than on allopatric fruit media $\left(F_{1,3}=1.11, P=0.37\right.$; Fig. 4D; Fig. S4D).

\section{Relative importance of adaptive phenotypic plasticity and local adaptation}

When analyzing G0 and G2 data together (Fig. 5B), our new statistical method showed that the higher preference for sympatric fruit media had a significant genetic origin $\left(\right.$ Fgenetic $_{1,3}=$ 
$12.77, P=0.037$ with a positive estimate of SAgeneticG0G2; Table S7). Overall, local adaptation (SAgenetic SOG $_{2}$ ) explained $74.6 \%$ of the variance of the genetic interaction between the test and original environments in G0 and G2. While phenotypic plasticity was in the maladaptive direction (negative estimate of SAplasticGo; Table S7), it was not significantly different from zero (Fplastic $1,3=3.54, P=0.16)$.

For oviposition stimulation, the absence of pattern in G0 and G2 when analyzed independently was confirmed, as no significant genetic or plastic effects were detected when analyzing G0 and G2 data together (Fig. 5C-D, Table S7; Fgenetic $1,3=2.26, P=0.23$ and

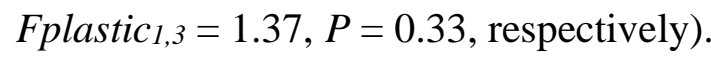

Higher performance on sympatric fruit media (Fig. 5F) had a significant genetic origin (Fgenetic $1,3=37.68, P=0.009$, with a positive estimate of SAgenetic G1G3; Table S7). Overall, local adaptation (SAgenetic SIG3 $_{3}$ ) explained $90.2 \%$ of the variance of the genetic interaction between the test and original environments in both G1 and G3. While phenotypic plasticity was in the adaptive direction (positive estimate of SAplasticGl; Table S7), it was not significantly different from zero (Fplastic $1,3=2.16, P=0.24)$.

\section{Testing for correlations between female preference and offspring performance}

We tested for a correlation between oviposition preference and offspring performance, after controlling for potential confounding factors (Appendix S7). We found that oviposition preference in G0 was positively correlated with offspring performance in G1, and oviposition preference in G2 was positively correlated with offspring performance in G3 (Fig. S9). The correlation coefficient was significantly different from zero for the G2/G3 generation test, whereas the $95 \%$ confidence intervals of the correlation coefficient included zero for the G0/G1 generation test. 


\section{Discussion}

In the present study, we used natural populations of $D$. suzukii to evaluate the roles of adaptive phenotypic plasticity and local adaptation in driving differences in oviposition preference, oviposition stimulation and offspring performance between sympatric and allopatric fruits. We found a pattern of local adaptation for oviposition preference and offspring performance, but not for oviposition stimulation. Females preferred their sympatric fruits, and their offspring performed better on those fruits. We did not find a signature of adaptive phenotypic plasticity. If it plays a role in the fit to the environment, that role is small enough to be masked by the effects of non-adaptive plasticity and local adaptation.

\section{Strong variation in quality among populations in both sympatric and allopatric environments}

For each of the three traits, we found consistent correlations among populations between sympatric and allopatric fruits: some populations had the strongest preference or performance across tested environments, highlighting the high variation in intrinsic quality among populations. This variability across populations has several possible sources. First, the high variation in quality among populations may be related to their genetic characteristics (e.g., variation in genetic load). Second, even when populations evolve on the same fruit, the number of generations spent on the host fruit might vary across populations, which could result in different levels of adaptation. Third, even if the number of generations on the same fruit was the same, populations might vary in the distance of their ancestral founding population to the phenotypic optima of the different fruits (Gómez et al., 2009). All else being equal, populations that started further away from the optima will be less adaptated than those that started closer to the optimum. 


\section{The higher preference and performance in sympatric fruit media is driven by local adaptation rather than adaptive phenotypic plasticity}

We found that the stronger oviposition preference and higher performance in sympatry than in allopatry was due to genetic and not plastic responses. Our research alters current understanding of how environmental grain shapes how organisms fit their environments. It appears that, at least in large diverse populations, local adaptation evolves rapidly in intermediate grained environments. Based on average temperatures in the studied areas, the period of availability of each host fruit and the generation time estimates in the laboratory (Poyet et al., 2015; Olazcuaga et al., 2019), our results suggest that local adaptation can evolve in less than four generations in natural populations of D. suzukii. This indicates that the evolutionary process of local adaptation, including lower relative fitness in allopatric environments, can be more rapid than traditionally thought (e.g., 10 generations in natural populations of $D$. melanogaster; Bergland et al., 2008). In agreement with this, a rapid adaptation over only four generations has been recently documented in D. melanogaster populations evolving in semi-natural conditions (Rudman et al., 2021). As populations often switch hosts, the rapid and dynamic process of local adaptation in D. suzukii seems to be transient. Transient and rapid local adaptation has also been seen in experimental populations of Tetranychus urticae in a heterogeneous environment (Bisschop et al., 2019). The seasonality of host fruits might result in balancing selection at adaptive loci that likely maintain high standing genetic variation in natural $D$. suzukii populations at and around those loci. The fast genetic adaptation we observed could be favored by the large effective size of D. suzukii populations and by the strong and divergent selective pressure exerted by seasonal fruits. The population size of $D$. suzukii in the studied area is likely to be very large (on the order of $10^{4}$, according to estimates in American cherry orchards; Tochen et al., 2014; Wiman et al., 2014). The high levels of genetic diversity observed in D. suzukii populations sampled 
in the South of France (Fraimout et al., 2017; Olazcuaga et al., 2020) are in agreement with these estimates. Differences in chemical composition among fruits likely result in strong pressures on traits such as offspring performance, as evidenced by a recent experimental evolution study (Olazcuaga et al., 2021).

Although our analysis method is based on the presence of genetic basis of traits measured before and after a common environment, we did not detect correlations between G0 (G1) and G2 (G3) generations. This inconsistency can be explained by the presence of nonadaptive plasticity (Fig. 1C), which can mask the genetic effects responsible for local adaptation patterns. In addition, we did not detect adaptive phenotypic plasticity although we had enough statistical power. This lack of adaptive phenotypic plasticity in this context may seem surprising at first sight because Drosophila species, including D. suzukii, usually excel at using cues to optimize their oviposition behavior (Jaenike, 1983; Papaj \& Rausher, 1983; Little \& Ebert, 2000). This absence of adaptive phenotypic plasticity suggests that in the case of $D$. suzukii the changes in plant hosts are not frequent enough for plasticity to be strongly beneficial. In addition, the flies were sampled in an agricultural region in France with a low level of polycultural practice, which might also explain this lack of plasticity, as different fruits are rarely available at the same time within the same area.

\section{Relationships between female oviposition preference and offspring performance}

We found that oviposition preference in generation G2 was positively and significantly correlated with offspring performance in generation G3. Previous studies that tested for this correlation in D. suzukii have shown mixed results (Poyet et al., 2015; Olazcuaga et al., 2019; Shu et al., 2021b). Our results based on natural populations of D. suzukii suggest that selection pressures on oviposition preference might be a driver of host fruit adaptation and could speed up local adaptation. This is consistent with previous theoretical and experimental studies showing that selective pressures on oviposition preference could feedback positively 
on the evolution of performance for local hosts (Whitlock, 1996; Berlocher \& Feder, 2002;

Via \& Hawthorne, 2002). In particular, if preference and performance traits are genetically linked, strong selection pressures on oviposition preference, at least for the three fruits studied here, could lead to the evolution of performance traits (Wood et al., 1999; Berner \& ThibertPlante, 2015).

\section{Limits of our experimental approach}

Our experiments do not get rid of the common drawback of trying to infer the fitness of natural populations using experiments in the lab and not in natural conditions (Reznick \& Ghalambor, 2005). First, the small scale spatial structure of our lab experimental device for studying the choice among multiple fruits deviates considerably from the conditions and geographic scales found in natural landscapes and may therefore provide an incomplete picture of the adaptive processes regarding oviposition preference in D. suzukii. Second, the yeasts and bacteria in fruits represent an important source of protein for the egg-to-adult survival of D. suzukii (Lewis \& Hamby, 2019; Bing et al., 2021). Moreover, the presence of microbes has been shown to modify the foraging behavior of $D$. suzukii females (Shu et al., 2021a), their oviposition preference and offspring performance (Bellutti et al., 2018).

Therefore, if the microbial community differs among cherry, strawberry and blackberry, our results could be at least partly explained by the nutritional effect of a fly microbiome transmitted over generations rather than to a genetic effect. Experiments in D. melanogaster have shown that the bacterial community could be transmitted over successive generations in the laboratory (Téfit et al., 2018) and that both Acetobacter and Lactobacilli can grow in media with concentration of antimicrobials similar to the one in our media (Obadia et al., 2018). Using axenic individuals could help testing for the role of the microbiome in the patterns we detected, although the absence of microbiota itself could have an important impact on both preference and performance. 


\section{Conclusion and perspectives}

We studied oviposition preference, oviposition stimulation and offspring performance in $D$. suzukii to evaluate the relative influence of genetic and plastic effects on the match between phenotypic traits and the local environment, here the host fruit. We found a pattern of local adaptation for oviposition preference and offspring performance, but not for oviposition stimulation. We found no evidence of adaptive phenotypic plasticity. Our study hence demonstrates that spatially and temporally variable selection does not prevent the rapid evolution of local adaptation in natural populations over a short number of generations. This dynamic process appears to be transient as populations rapidly adapt to new host fruits as they become available throughout the year. In addition to testing for the potential role of the microbiome in the pattern we detected, a stimulating follow up to our study would be to identify the physiological pathways under selection using genome-environment association study based on populations sampled from different host plants. Hopefully, such a combination of statistical, molecular, and quantitative approaches will provide useful insights into the genomic and phenotypic responses to divergent selection among host fruits in phytophagous generalist insects. Finally, our new statistical method will likely contribute to the quantification of the relative contribution of local adaptation and adaptive phenotypic plasticity in any other organisms experiencing spatially or temporally variable selection. 


\section{Acknowledgements}

We are grateful to V. Ravigné and B. Facon for insightful discussions, P. Audiot, M.P.

Chapuis, L. Benoit, N. Vieira, J. Froissard, A. L. Clamens, S. Nidelet and L. Sauné for technical assistance, S. Bonamour for her comments on the manuscript, V. Cazalis and R. Patin for their help with drawing the map, and to the Sicoly cooperative for providing us with some fruit purees. L.O., M.G., J.F. and A.E. were supported by the Languedoc-Roussillon region (France) through the European Union program FEDER FSE IEJ 2014-2020 (project CPADROL) and the INRAE scientific department SPE (AAP-SPE 2016). R.A.H. acknowledges support from the National Science Foundation (DEB-0949619), USDA Agriculture and Food Research Initiative award (2014-67013-21594), Hatch project 1012868, the French Agropolis Fondation (LabEx Agro-Montpellier) through the AAP "International Mobility" (CfP 2015-02), the French programme investissement d'avenir, and the LabEx CEMEB through the AAP "invited scientist 2016". N.O.R. acknowledges support from the CeMEB LabEx/University of Montpellier (ANR-10-LABX-04-01) and the INRAE scientific department SPE (AAP-SPE 2021PestAdapt).

\section{Author contributions}

Conceptualization and funding acquisition: L.O., J.F., M.G., R.A.H., N.O.R. and A.E.;

Statistical analyses and writing: L.O. with inputs of J.F., M.G., R.A.H., N.O.R. and A.E.;

Data acquisition and field sampling: L.O., C.D., A.L., J-L.I., R.V., R.G., C.S. and A.E.

\section{Data accessibility}

The data and R scripts for our analyses are available at: https://github.com/nrode/NatPop2021

They will be archived on Dryad upon acceptance of the manuscript. 
bioRxiv preprint doi: https://doi.org/10.1101/2022.03.01.482503; this version posted March 4, 2022. The copyright holder for this preprint (which was not certified by peer review) is the author/funder, who has granted bioRxiv a license to display the preprint in perpetuity. It is made available under aCC-BY-NC-ND 4.0 International license.

\section{Conflict of interest}

The authors declare no conflict of interest. 


\section{References}

Aly. (2018) Laboratory host selection and development of immature Drosophila suzukii (Diptera: Drosophilidae) on fruits and artificial diets. Journal of Phytopathology and Pest Management, 5, 129-142.

Auld, J.R., Agrawal, A.A. \& Relyea, R.A. (2010) Re-evaluating the costs and limits of adaptive phenotypic plasticity. Proceedings of the Royal Society B: Biological Sciences, 277, $503-511$.

Barghi, N., Hermisson, J. \& Schlötterer, C. (2020) Polygenic adaptation: a unifying framework to understand positive selection. Nature Reviews Genetics, 21, 769-781.

Bellutti, N., Gallmetzer, A., Innerebner, G., Schmidt, S., Zelger, R. \& Koschier, E.H. (2018) Dietary yeast affects preference and performance in Drosophila suzukii. Journal of Pest Science, 91, 651-660.

Bergland, A.O., Genissel, A., Nuzhdin, S.V.\& Tatar, M. (2008) Quantitative Trait Loci Affecting Phenotypic Plasticity and the Allometric Relationship of Ovariole Number and Thorax Length in Drosophila melanogaster. Genetics, 180, 567-582.

Berlocher, S.H. \& Feder, J.L. (2002) Sympatric speciation in phytophagous insects: moving beyond controversy? Annual review of entomology, 47, 773-815.

Berner, D. \& Thibert-Plante, X. (2015) How mechanisms of habitat preference evolve and promote divergence with gene flow. Journal of evolutionary biology, 28, 1641-1655.

Bing, X.-L., Winkler, J., Gerlach, J., Loeb, G. \& Buchon, N. (2021) Identification of natural pathogens from wild Drosophila suzukii. Pest Management Science, 77, 1594-1606.

Bisschop, K., Mortier, F., Etienne, R.S. \& Bonte, D. (2019) Transient local adaptation and source-sink dynamics in experimental populations experiencing spatially heterogeneous environments. Proceedings of the Royal Society B: Biological Sciences, 286, 20190738.

Blanquart, F., Kaltz, O., Nuismer, S.L. \& Gandon, S. (2013) A practical guide to measuring local adaptation. Ecology Letters, 16, 1195-1205.

Bonnet, X., Brischoux, F., Briand, M. \& Shine, R. (2021) Plasticity matches phenotype to local conditions despite genetic homogeneity across 13 snake populations. Proceedings of the Royal Society B: Biological Sciences, 288, 20202916.

Botero, C.A., Weissing, F.J., Wright, J. \& Rubenstein, D.R. (2015) Evolutionary tipping points in the capacity to adapt to environmental change. Proceedings of the National Academy of Sciences, 112, 184-189.

Bradshaw, A.D. (1965) Evolutionary significance of phenotypic plasticity in plants. In 
Advances in Genetics. Academic Press, pp. 115-155.

Burrack, H.J., Fernandez, G.E., Spivey, T. \& Kraus, D.A. (2013) Variation in selection and utilization of host crops in the field and laboratory by Drosophila suzukii Matsumara (Diptera: Drosophilidae), an invasive frugivore. Pest Management Science, 69, 1173-1180.

Chevin, L.-M., Lande, R. \& Mace, G.M. (2010) Adaptation, plasticity, and extinction in a changing environment: towards a predictive theory. PLOS Biology, 8, e1000357.

Chiu, J.C., Jiang, X., Zhao, L., Hamm, C.A., Cridland, J.M., Saelao, P., et al. (2013) Genome of Drosophila suzukii, the spotted wing drosophila. G3 (Bethesda, Md.), 3, 2257-71.

DeWitt, T.J. \& Scheiner, S.M. (2004) Phenotypic plasticity: functional and conceptual approaches. OUP USA, Oxford; New York.

Dewitt, T.J., Sih, A. \& Wilson, D.S. (1998) Costs and limits of phenotypic plasticity. Trends in Ecology \& Evolution, 13, 77-81.

Dury, G. \& Wade, M. (2019) When mother knows best: A population genetic model of transgenerational versus intragenerational plasticity. Journal of Evolutionary Biology, 33.

Edelaar, P., Jovani, R. \& Gomez-Mestre, I. (2017) Should I change or should I go? Phenotypic plasticity and matching habitat choice in the adaptation to environmental heterogeneity. The American Naturalist, 190, 506-520.

Enbody, E.D., Pettersson, M.E., Sprehn, C.G., Palm, S., Wickström, H. \& Andersson, L. (2021) Ecological adaptation in European eels is based on phenotypic plasticity. Proceedings of the National Academy of Sciences, 118, e2022620118.

Endler, J.A. (1977) Geographic Variation, Speciation and Clines. (MPB-10), Volume 10. Princeton University Press.

Fraimout, A., Debat, V., Fellous, S., Hufbauer, R.A., Foucaud, J., Pudlo, P., et al. (2017) Deciphering the routes of invasion of Drosophila suzukii by means of ABC random forest. Molecular Biology and Evolution, 34, 980-996.

Fry, J.D. (1996) The evolution of host specialization: are trade-offs overrated? The American Naturalist, 148, 84-107.

García-Ramos, G. \& Kirkpatrick, M. (1997) Genetic Models of Adaptation and Gene Flow in Peripheral Populations. Evolution, 51, 21-28.

Gavrilets, S. \& Scheiner, S.M. (1993) The genetics of phenotypic plasticity. V. Evolution of reaction norm shape. Journal of Evolutionary Biology, 6, 31-48.

Ghalambor, C.K., McKay, J.K., Carroll, S.P. \& Reznick, D.N. (2007) Adaptive versus non-adaptive phenotypic plasticity and the potential for contemporary adaptation in new environments. Functional Ecology, 21, 394-407. 
Gillespie, J. (1974) The role of environmental grain in the maintenance of genetic variation. The American Naturalist, 108, 831-836.

Gómez, J.M., Abdelaziz, M., Camacho, J.P.M., Muñoz-Pajares, A.J. \& Perfectti, F. (2009) Local adaptation and maladaptation to pollinators in a generalist geographic mosaic. Ecology Letters, 12, 672-682.

Gotthard, K. \& Nylin, S. (1995) Adaptive plasticity and plasticity as an adaptation: a selective review of plasticity in animal morphology and life history. Oikos, 74, 3-17.

Hansen, T.F., Carter, A.J.R. \& Pélabon, C. (2006) On Adaptive Accuracy and Precision in Natural Populations. The American Naturalist, 168, 168-181.

Hauser, M. (2011) A historic account of the invasion of Drosophila suzukii (Matsumura) (Diptera: Drosophilidae) in the continental United States, with remarks on their identification. Pest Management Science, 67, 1352-1357.

Hereford, J. (2009) A quantitative survey of local adaptation and fitness trade-offs. The American Naturalist, 173, 579-588.

Jaenike, J. (1978) On optimal oviposition behavior in phytophagous insects. Theoretical Population Biology, 14, 350-356.

Jaenike, J. (1983) Induction of host preference in Drosophila melanogaster. Oecologia, 58, 320-325.

Jong, G.D. (1999) Unpredictable selection in a structured population leads to local genetic differentiation in evolved reaction norms. Journal of Evolutionary Biology, 12, 839851.

Kawecki, T.J. \& Ebert, D. (2004) Conceptual issues in local adaptation. Ecology Letters, 7, 1225-1241.

Kenis, M., Tonina, L., Eschen, R., Sluis, B. van der, Sancassani, M., Mori, N., et al. (2016) Non-crop plants used as hosts by Drosophila suzukii in Europe. Journal of Pest Science, 89, 735-748.

Lande, R. (1976) Natural selection and random genetic drift in phenotypic evolution. Evolution, 314-334.

Lande, R. (2009) Adaptation to an extraordinary environment by evolution of phenotypic plasticity and genetic assimilation. Journal of Evolutionary Biology, 22, 14351446.

Lande, R. (2015) Evolution of phenotypic plasticity in colonizing species. Molecular Ecology, 24, 2038-2045.

Lee, J.C., Bruck, D.J., Dreves, A.J., Ioriatti, C., Vogt, H. \& Baufeld, P. (2011) In Focus: 
Spotted wing drosophila, Drosophila suzukii, across perspectives. Pest Management Science, 67, 1349-1351.

Levins, R. (1968) Evolution in changing environments: some theoretical explorations. Princeton University Press.

Lewis, M.T. \& Hamby, K.A. (2019) Differential impacts of yeasts on feeding behavior and development in larval Drosophila suzukii (Diptera:Drosophilidae). Scientific Reports, 9, $1-12$.

Little, T.J. \& Ebert, D. (2000) The cause of parasitic infection in natural populations of Daphnia (Crustacea: Cladocera): the role of host genetics. Proceedings of the Royal Society of London. Series B: Biological Sciences, 267, 2037-2042.

McDonald, J.H. (2014) Handbook of Biological Statistics (3rd ed.). Sparky House Publishing, Baltimore, Maryland.

Merila, J. \& Hendry, A.P. (2014) Climate change, adaptation, and phenotypic plasticity: the problem and the evidence. Evolutionary Applications, 7, 1-14.

Miller, R.G. (1997) Beyond ANOVA: basics of applied statistics. Chapman and Hall/CRC.

Obadia, B., Keebaugh, E.S., Yamada, R., Ludington, W.B. \& Ja, W.W. (2018) Diet influences host-microbiota associations in Drosophila. Proceedings of the National Academy of Sciences of the United States of America, 115, E4547-E4548.

Olazcuaga, L., Foucaud, J., Gautier, M., Deschamps, C., Loiseau, A., Leménager, N., et al. (2021) Adaptation and correlated fitness responses over two time scales in Drosophila suzukii populations evolving in different environments. Journal of Evolutionary Biology, 34, $1225-1240$.

Olazcuaga, L., Loiseau, A., Parrinello, H., Paris, M., Fraimout, A., Guedot, C., et al. (2020) A Whole-Genome Scan for Association with Invasion Success in the Fruit Fly Drosophila suzukii Using Contrasts of Allele Frequencies Corrected for Population Structure. Molecular Biology and Evolution, 37, 2369-2385.

Olazcuaga, L., Rode, N.O., Foucaud, J., Facon, B., Ravigné, V., Ausset, A., et al. (2019) Oviposition Preference and Larval Performance of Drosophila suzukii (Diptera: Drosophilidae), Spotted-Wing Drosophila: Effects of Fruit Identity and Composition. Environmental Entomology, 48, 867-881.

Papaj, D.R. \& Rausher, M.D. (1983) Chap 3. Individual variation in host location by phytophagous insects. In Herbivorous Insects: Host-seeking Behavior and mechanisms. Elsevier. 
Pigliucci, M. (2001) Phenotypic plasticity: beyond nature and nurture. JHU Press.

Pigliucci, M., Murren, C.J. \& Schlichting, C.D. (2006) Phenotypic plasticity and evolution by genetic assimilation. Journal of Experimental Biology, 209, 2362-2367.

Poyet, M., Le Roux, V., Gibert, P., Meirland, A., Prevost, G., Eslin, P., et al. (2015) The wide potential trophic niche of the asiatic fruit fly Drosophila suzukii: The key of its invasion success in temperate Europe? PLoS ONE, 10.

Price, T.D., Qvarnström, A. \& Irwin, D.E. (2003) The role of phenotypic plasticity in driving genetic evolution. Proceedings of the Royal Society of London. Series B: Biological Sciences, 270, 1433-1440.

R Core Team. (2014) R: A language environment for statistical computing.

Rago, A., Kouvaris, K., Uller, T. \& Watson, R. (2019) How adaptive plasticity evolves when selected against. PLOS Computational Biology, 15, e1006260.

Rausher, M.D. (1988) Plant-Insect Interfaces. Ecology, 69, 295-296.

Ravigné, V., Dieckmann, U. \& Olivieri, I. (2009) Live where you thrive: joint evolution of habitat choice and local adaptation facilitates specialization and promotes diversity. The American Naturalist, 174, E141-E169.

Reznick, D.N. \& Ghalambor, C.K. (2005) Selection in nature: experimental manipulations of natural populations. Integrative and Comparative Biology, 45, 456-462.

Robinson, B.W. \& Dukas, R. (1999) The influence of phenotypic modifications on evolution: the Baldwin effect and modern perspectives. Oikos, 85, 582-589.

Rudman, S.M., Greenblum, S.I., Rajpurohit, S., Betancourt, N.J., Hanna, J., Tilk, S., et al. (2021) Direct observation of adaptive tracking on ecological timescales in Drosophila.

Scheiner, S.M. (2016) Habitat choice and temporal variation alter the balance between adaptation by genetic differentiation, a jack-of-all-trades strategy, and phenotypic plasticity. The American Naturalist, 187, 633-646.

Schmid, B. (1992) Phenotypic variation in plants. Evolutionary trends in plants, 6, 4560.

Shu, R., Hahn, D.A., Jurkevitch, E., Liburd, O.E., Yuval, B. \& Wong, A.C.-N. (2021a) Sex-Dependent Effects of the Microbiome on Foraging and Locomotion in Drosophila suzukii. Frontiers in Microbiology, 12, 1094.

Shu, R., Uy, L. \& Wong, A.C.-N. (2021b) Nutritional phenotype underlines the performance trade-offs of Drosophila suzukii on different fruit diets. Current Research in Insect Science, 100026.

Slatkin, M. (1985) Gene Flow in Natural Populations. Annual Review of Ecology and 
Systematics, 16, 393-430.

Sun, S.-J., Catherall, A.M., Pascoal, S., Jarrett, B.J.M., Miller, S.E., Sheehan, M.J., et al. (2020) Rapid local adaptation linked with phenotypic plasticity. Evolution Letters, 4, 345359.

Téfit, M.A., Gillet, B., Joncour, P., Hughes, S. \& Leulier, F. (2018) Stable association of a Drosophila-derived microbiota with its animal partner and the nutritional environment throughout a fly population's life cycle. Journal of Insect Physiology, 106, 2-12.

Tochen, S., Dalton, D.T., Wiman, N., Hamm, C., Shearer, P.W. \& Walton, V.M. (2014) Temperature-related development and population parameters for Drosophila suzukii (Diptera: Drosophilidae) on cherry and blueberry. Environmental Entomology, 43, 501-510.

Torres Dowdall, J., Handelsman, C.A., Ruell, E.W., Auer, S.K., Reznick, D.N. \& Ghalambor, C.K. (2012) Fine-scale local adaptation in life histories along a continuous environmental gradient in Trinidadian guppies. Functional Ecology, 26, 616-627.

Tufto, J. (2000) The evolution of plasticity and nonplastic spatial and temporal adaptations in the presence of imperfect environmental cues. The American Naturalist, 156, $121-130$.

Tufto, J. (2015) Genetic evolution, plasticity, and bet-hedging as adaptive responses to temporally autocorrelated fluctuating selection: A quantitative genetic model: evolutionary responses to fluctuating selection. Evolution, 69, 2034-2049.

Turesson, G. (1922) The genotypical response of the plant species to the habitat. Hereditas, 3, 211-350.

Uller, T. (2008) Developmental plasticity and the evolution of parental effects. TREE, 33.

Van Buskirk, J. \& Steiner, U.K. (2009) The fitness costs of developmental canalization and plasticity. Journal of Evolutionary Biology, 22, 852-860.

Via, S. (1991) The genetic structure of host plant adaptation in a spatial patchwork: demographic variability among reciprocally transplanted pea aphid clones. Evolution, 45, $827-852$.

Via, S. \& Hawthorne, D.D.J. (2002) The genetic architecture of ecological specialization: correlated gene effects on host use and habitat choice in pea aphids. The American Naturalist, 159, 76-88.

Via, S. \& Lande, R. (1985) Genotype-environment interaction and the evolution of phenotypic plasticity. Evolution, 39, 505-522.

Waddington, C.H. (1953) Genetic Assimilation of an Acquired Character. Evolution, 7, 
bioRxiv preprint doi: https://doi org/10.1101/2022.03 01.482503; this version posted March 4, 2022. The copyright holder for this preprint (which was not certified by peer review) is the author/funder, who has granted bioRxiv a license to display the preprint in perpetuity. It is made available under aCC-BY-NC-ND 4.0 International license.

$118-126$.

Walsh, D.B., Bolda, M.P., Goodhue, R.E., Dreves, A.J., Lee, J.C., Bruck, D.J., et al. (2011) Drosophila suzukii (Diptera: Drosophilidae): invasive pest of ripening soft fruit expanding its geographic range and damage potential. Journal of Integrated Pest Management, 2, 1-7.

Whitlock, M.C. (1996) The red queen beats the jack-of-all-trades: the limitations on the evolution of phenotypic plasticity and niche breadth. The American Naturalist, 148.

Wiman, N.G., Walton, V.M., Dalton, D.T., Anfora, G., Burrack, H.J., Chiu, J.C., et al. (2014) Integrating Temperature-Dependent Life Table Data into a Matrix Projection Model for Drosophila suzukii Population Estimation. PLOS ONE, 9, e106909.

Wood, T.K., Tilmon, K.J., Shantz, A.B., Harris, C.K. \& Pesek, J. (1999) The role of host-plant fidelity in initiating insect race formation. Evolutionary Ecology Research, 317 332.

Yampolsky, L.Y., Schaer, T.M.M. \& Ebert, D. (2014) Adaptive phenotypic plasticity and local adaptation for temperature tolerance in freshwater zooplankton. Proceedings of the Royal Society B: Biological Sciences, 281, 20132744. 


\section{Figures}

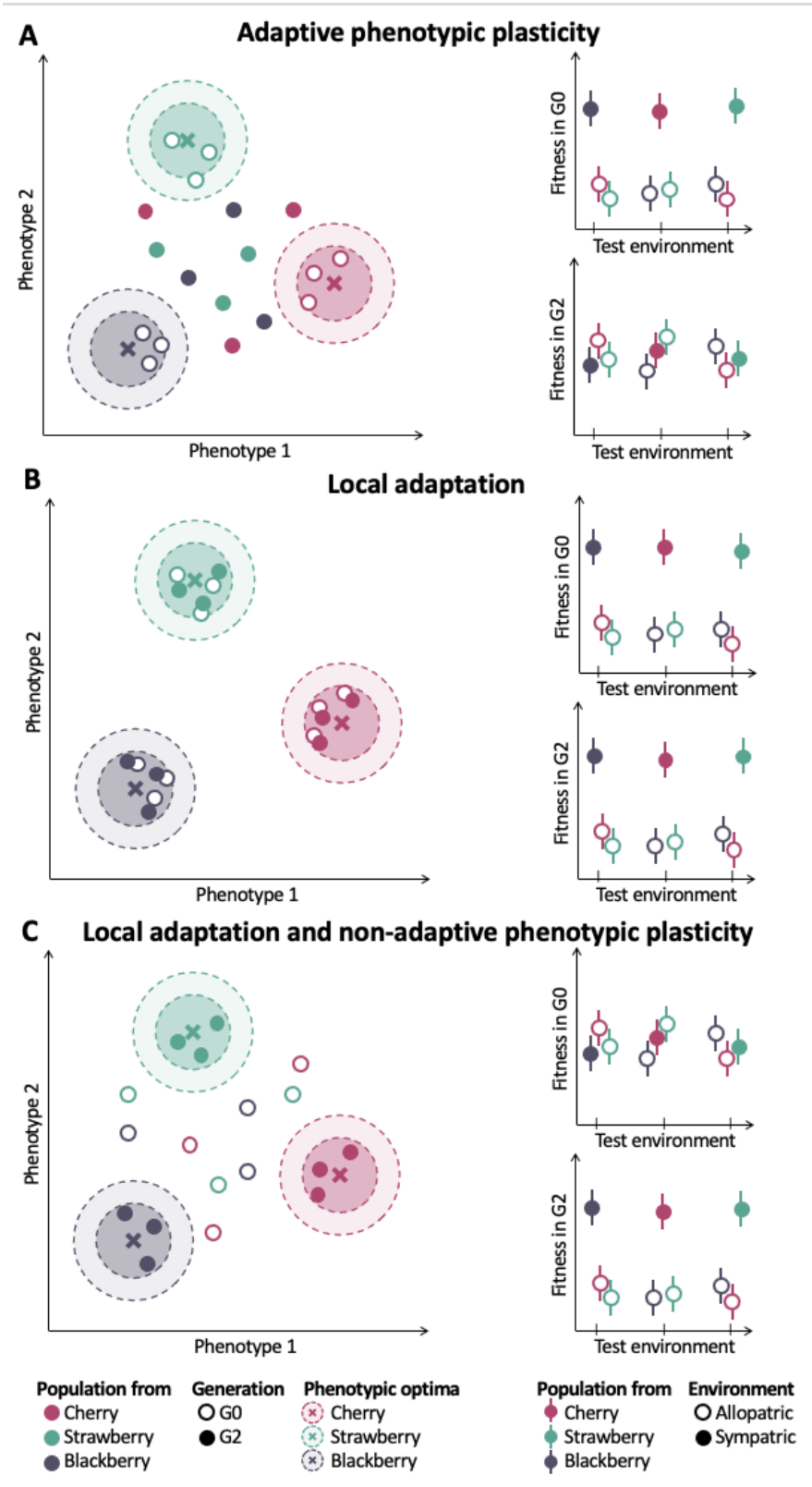

Figure 1. Theoretical predictions for (A) adaptive phenotypic plasticity, (B) local

adaptation, and (C) local adaptation with non-adaptive phenotypic plasticity. In the right panels, the two-dimensional fitness landscapes are represented. For each fruit, the position of the phenotypic optimum providing maximal fitness is represented by a cross. The positions of populations in G0 and G2 are represented by open and closed circles, respectively. The right panels provide the fitness measures in G0 and G2 with allopatric and sympatric measures represented by open and closed circles, respectively. 


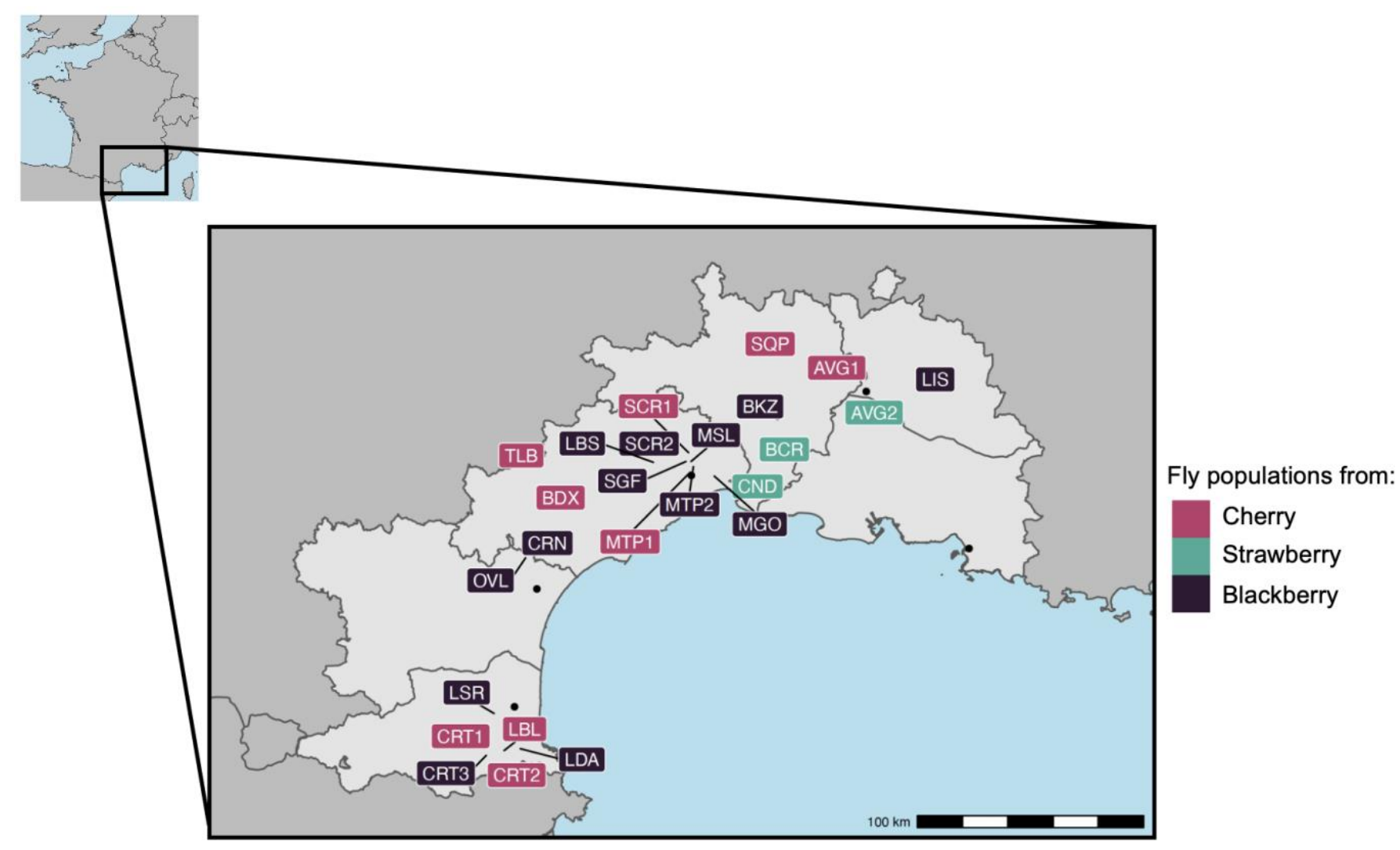

Figure 2. Geographic locations of the 25 sample sites where collected fruits (cherry, strawberry or blackberry) yielded enough $D$. suzukii adults in the lab to be included in this study. See Materials and Methods and Table S6 for details. 


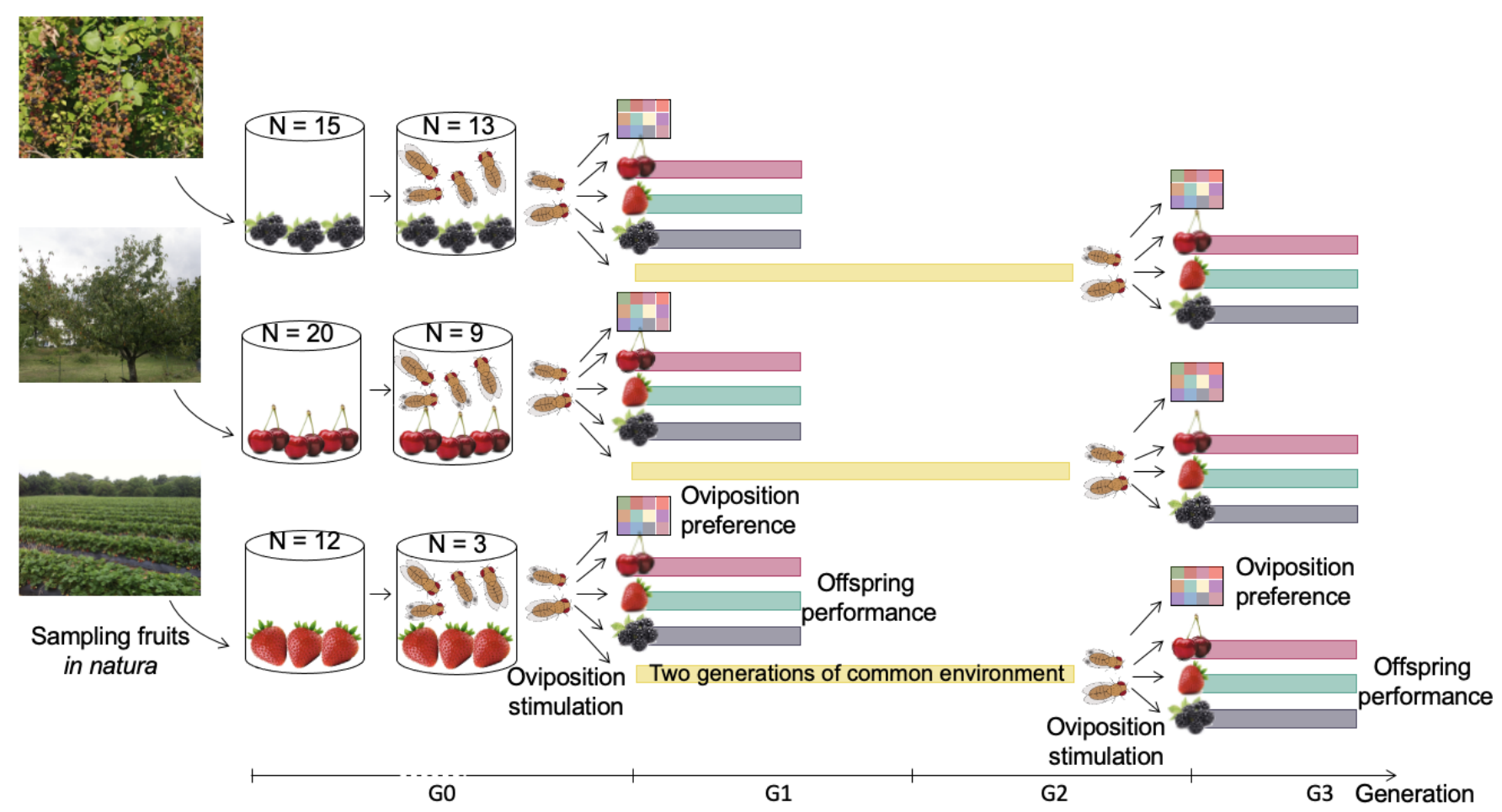

Figure 3. Graphical representation of the experimental design. A standard laboratory fly food medium instead of a fruit puree medium was used for the two generations of common environment. To test for potential temporal variability in experimental conditions, a control inbred D. suzukii line (WT3) was assayed together with each studied population (see main text for details). 

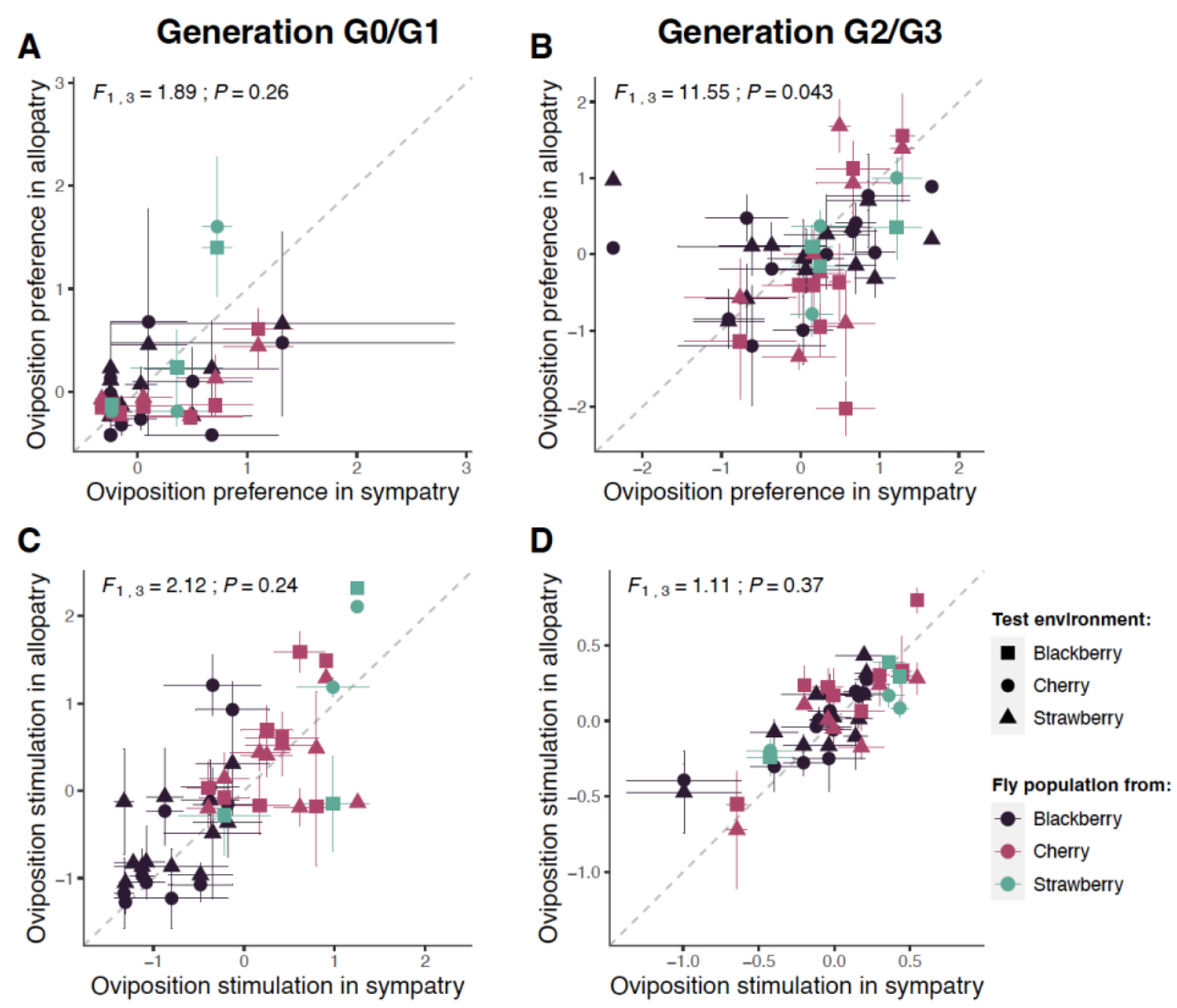

E

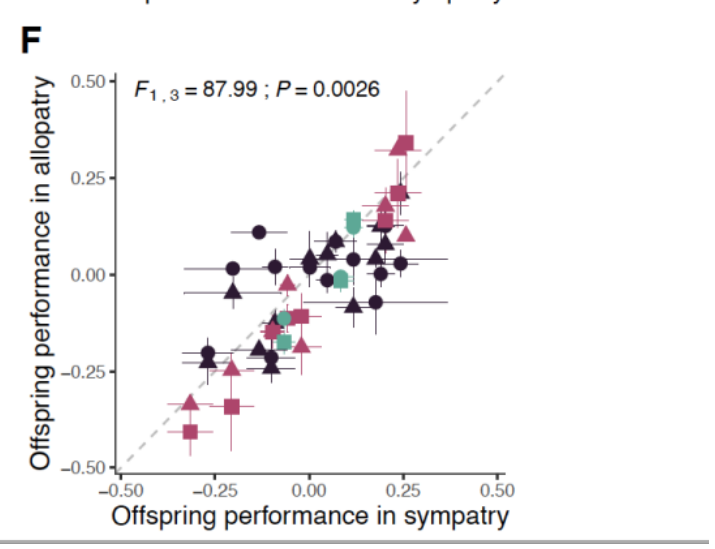

Figure 4. Relationship between the sympatric and allopatric fruit media for (A-B) oviposition preference, (C-D) oviposition stimulation, and (E-F) offspring performance in generations G0 or G1 (depending on the measured trait; left panels) and G2 or G3 (right panels). The mean of each population in each test environment is represented by a symbol whose shape and color depend on the test environment (fruit medium) and the fruit from which the population was collected in natura, respectively. The mean trait values are estimated using a linear model after controlling for variation in quality among fruit media, but also for variation in egg number among tubes for offspring performance. Error bars represent the standard error of mean estimates. Results ( $F$-statistics and $P$-values) from the $F$-test of Blanquart et al. (2013) are represented on each panel (see Appendix S3 for details). 

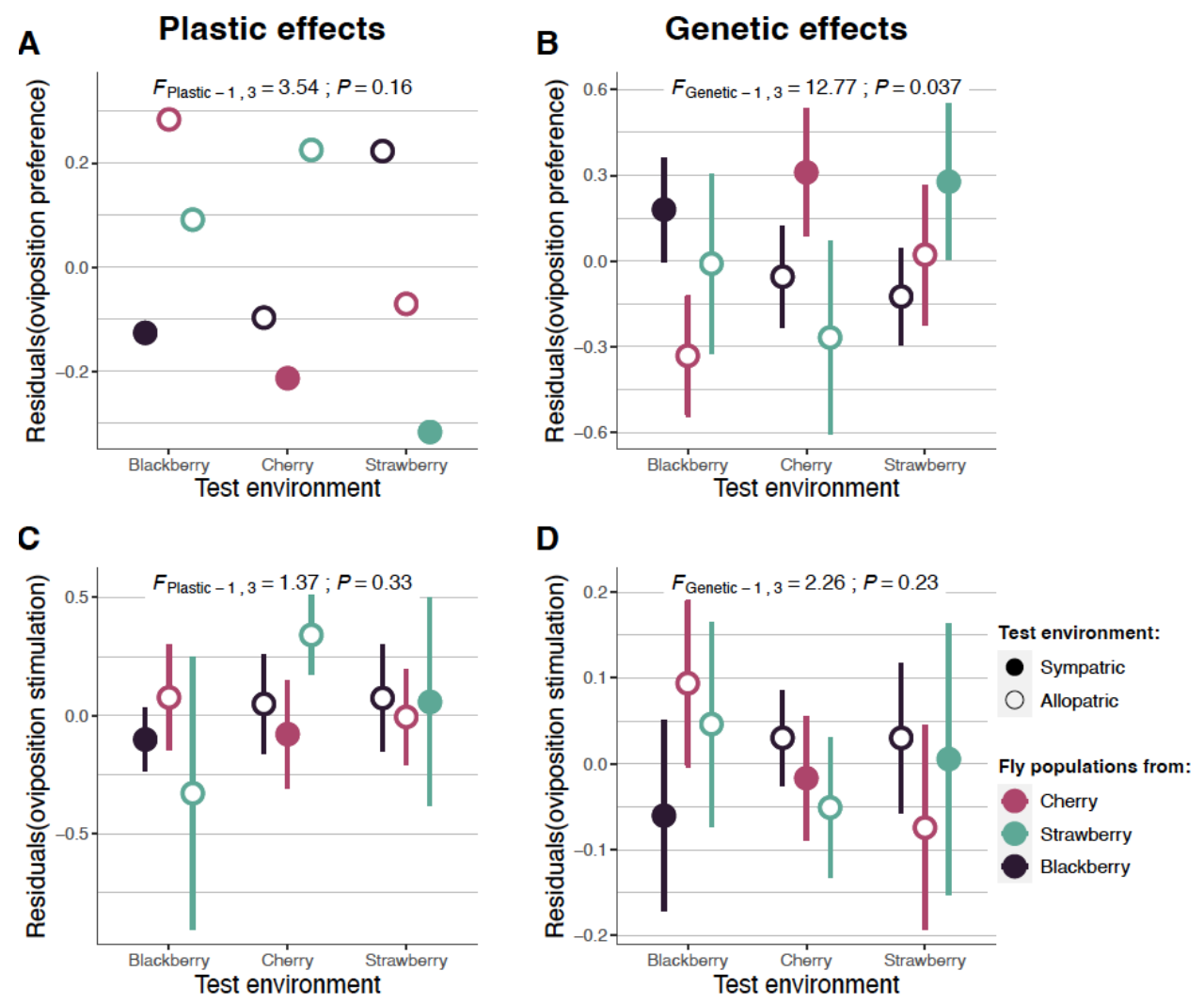

E
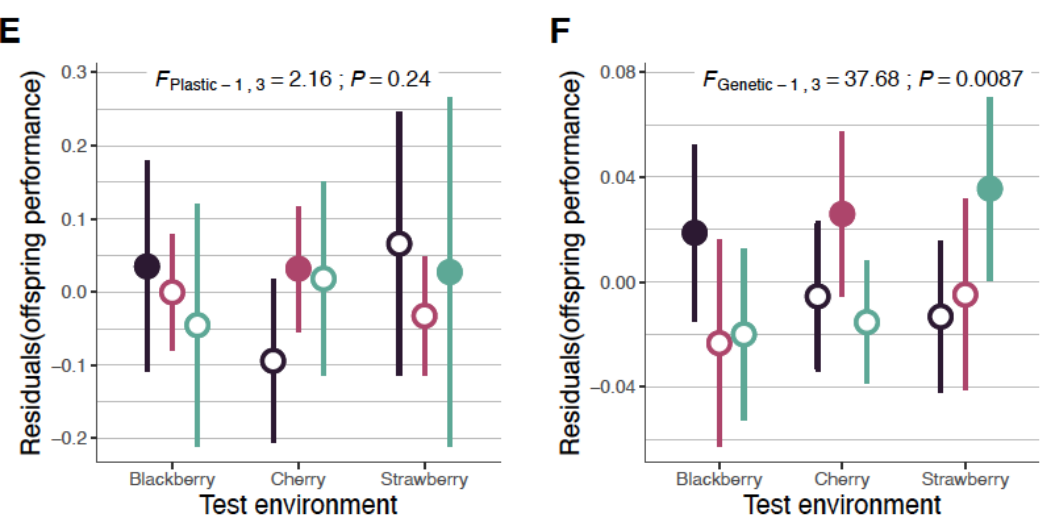

Figure 5. Average plastic (left panels) and genetic (right panels) effects across $D$. suzukii natural populations for (A-B) oviposition preference, (C-D) oviposition stimulation, and (E-F) offspring performance for each combination of original fruit environment and test

fruit environment. Results ( $F$-statistics and $P$-values) for the test of adaptive phenotypic plasticity (i.e., plastic effect) or local adaptation (i.e., genetic effects) are represented on each panel. The mean values of each trait across populations are represented for sympatric and allopatric combinations by closed and open circles, respectively $(n=9,3$ and 13 populations for cherry, strawberry and blackberry, respectively). Error bars represent $95 \%$ confidence intervals. For plastic effects in oviposition preference, $95 \%$ confidence intervals could not be computed due to a higher variance in $\mathrm{G} 2$ than in G0. 

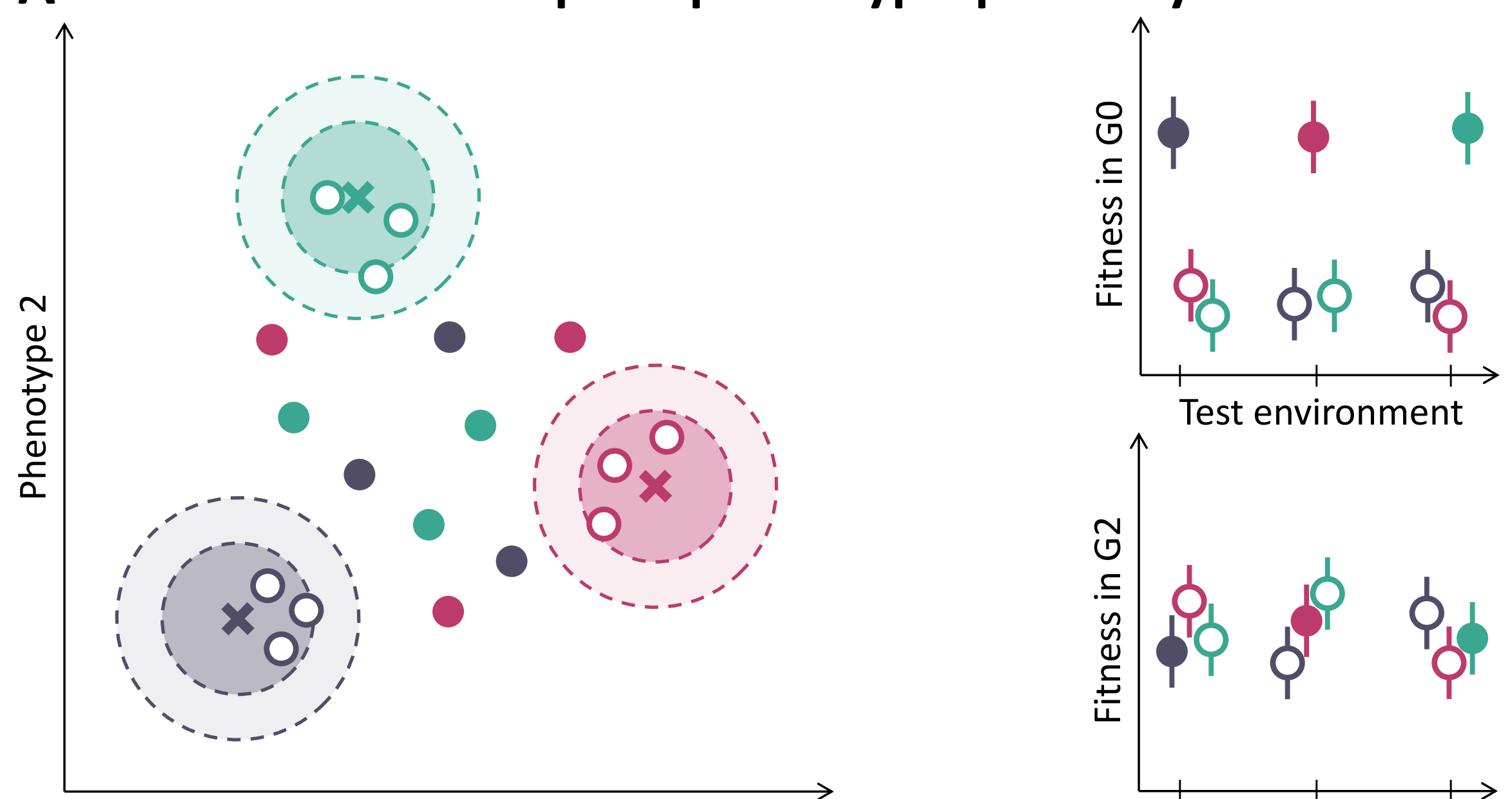

Phenotype 1

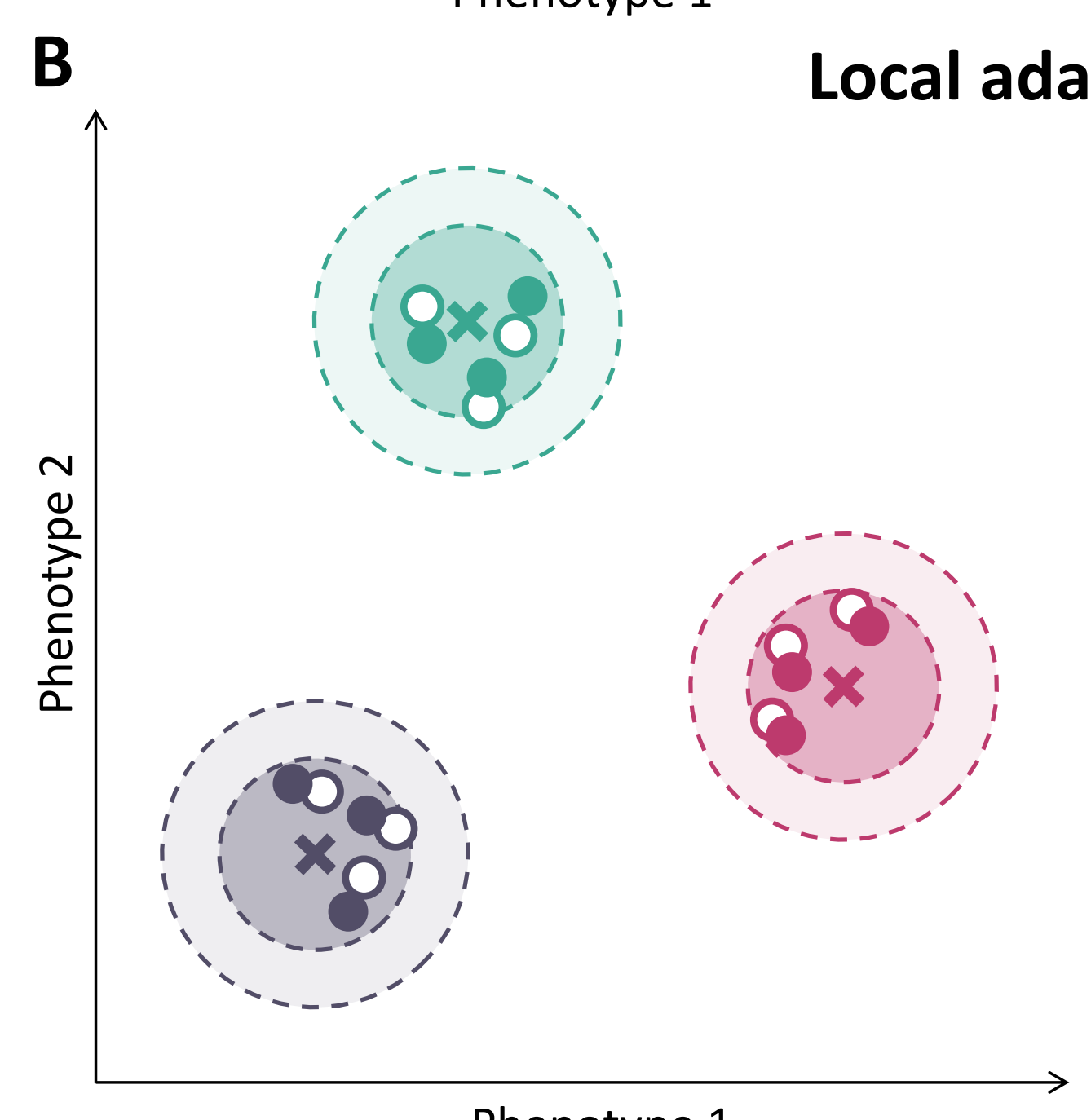

Phenotype 1
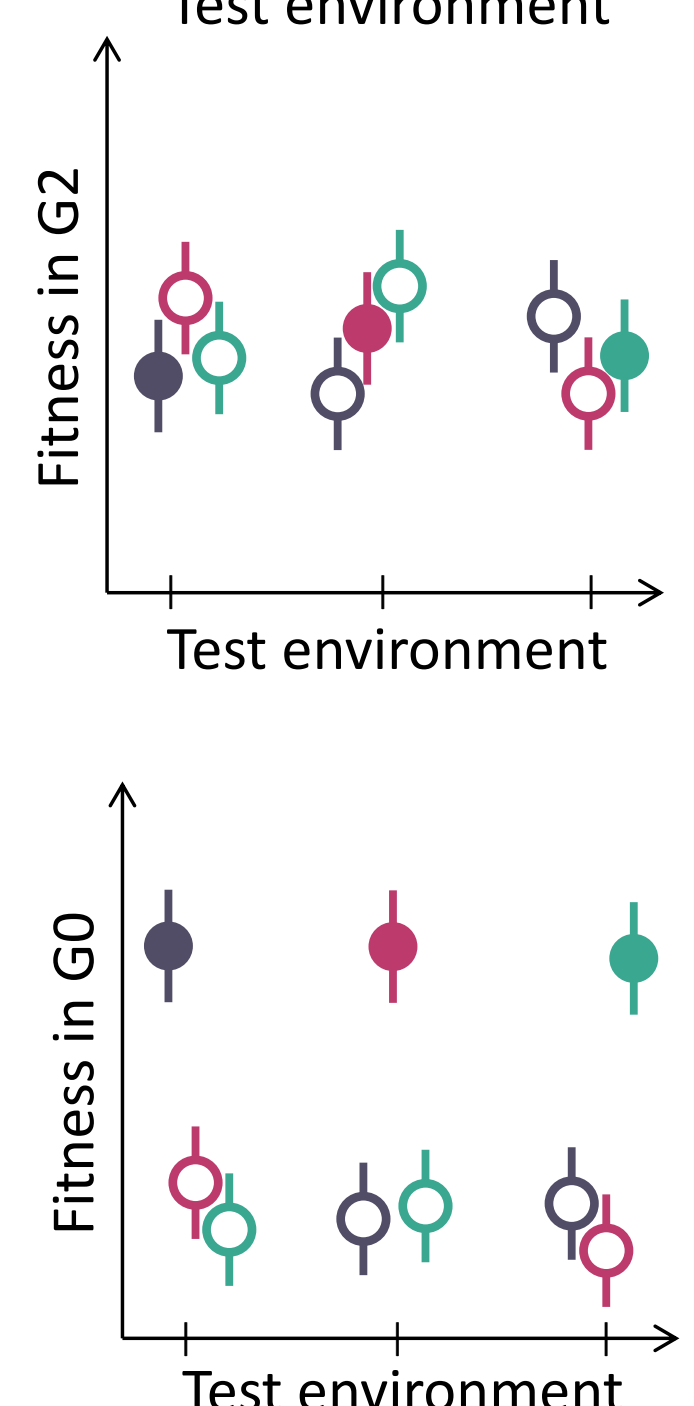

C Local adaptation and non-adaptive phenotypic plasticity

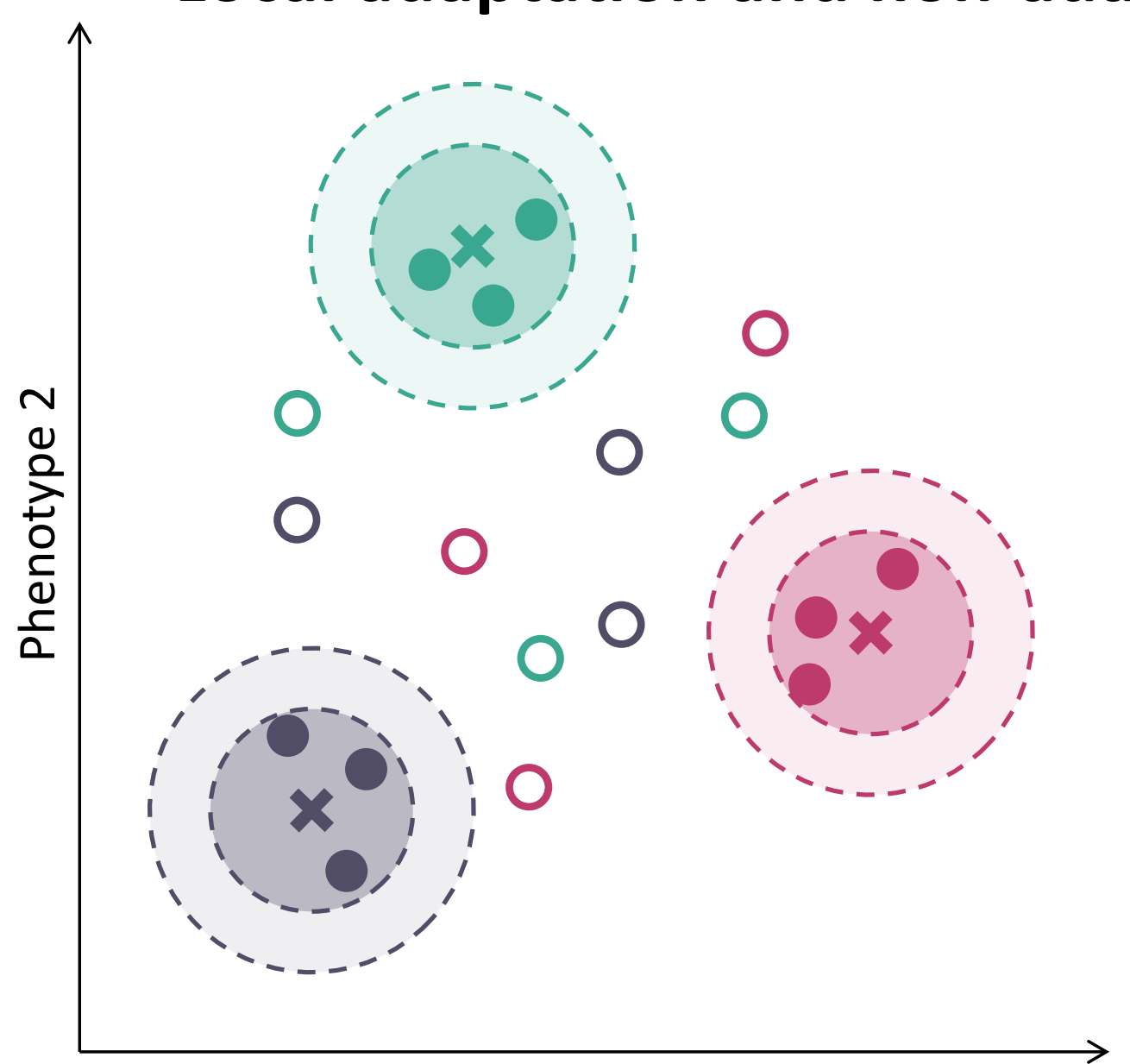

Phenotype 1
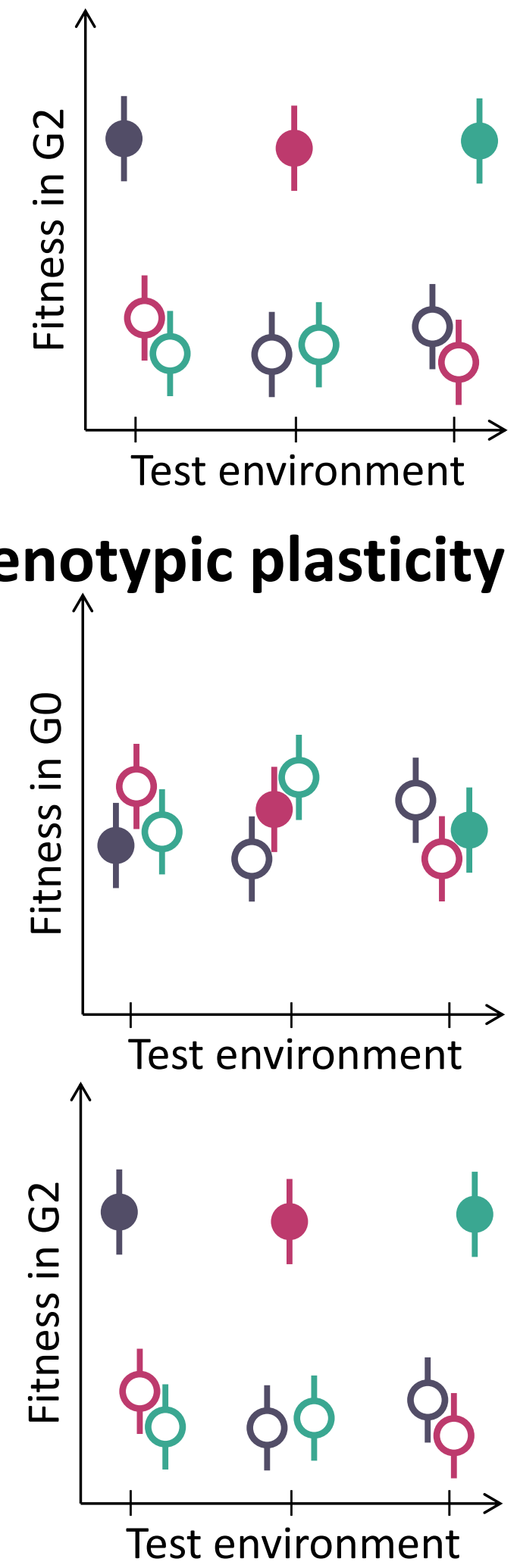

$\begin{array}{lll}\text { Population from } & \text { Generation } & \text { Phenotypic optima } \\ \text { Cherry } & \text { OG0 } & (x) \text { Cherry } \\ \text { Strawberry } & \text { OG2 } & \text { Strawberry } \\ \text { Blackberry } & & \text { S Backberry }\end{array}$

Population from Environment

Cherry OAllopatric

Strawberry Sympatric

Blackberry 


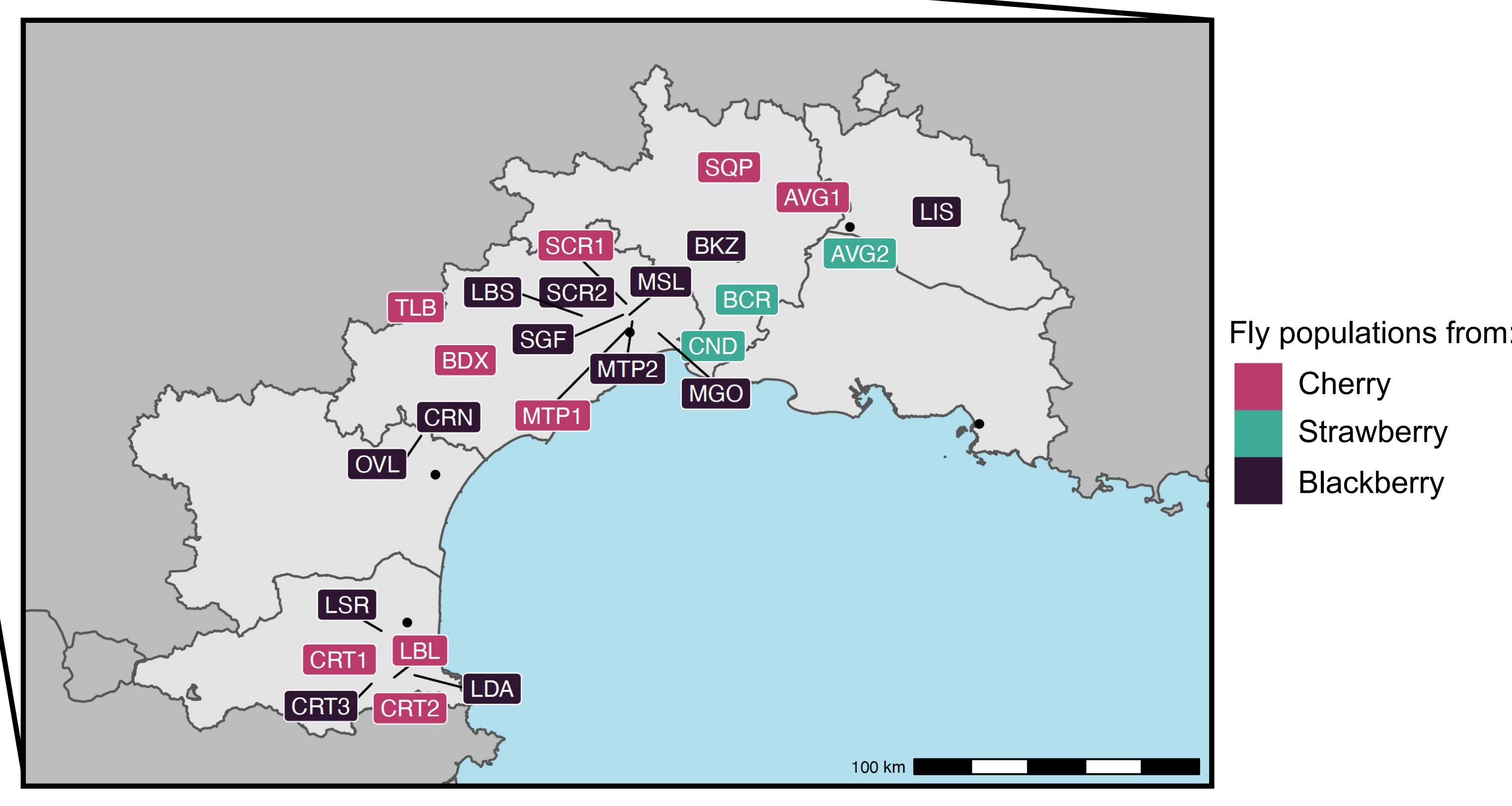


A

Generation G0/G1

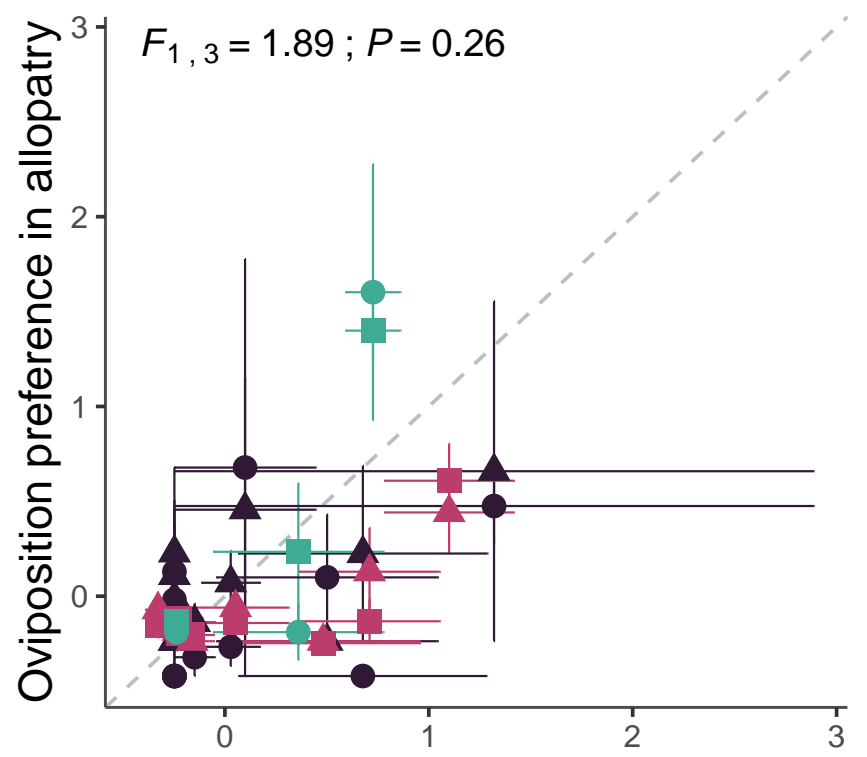

Oviposition preference in sympatry

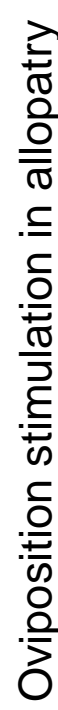

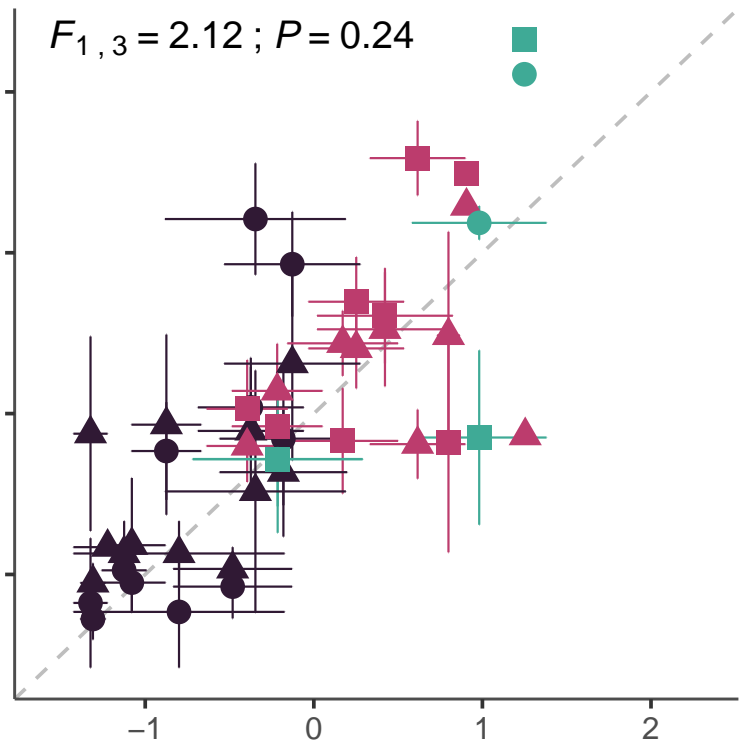

Oviposition stimulation in sympatry

E

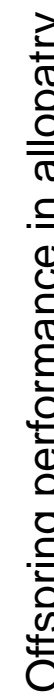

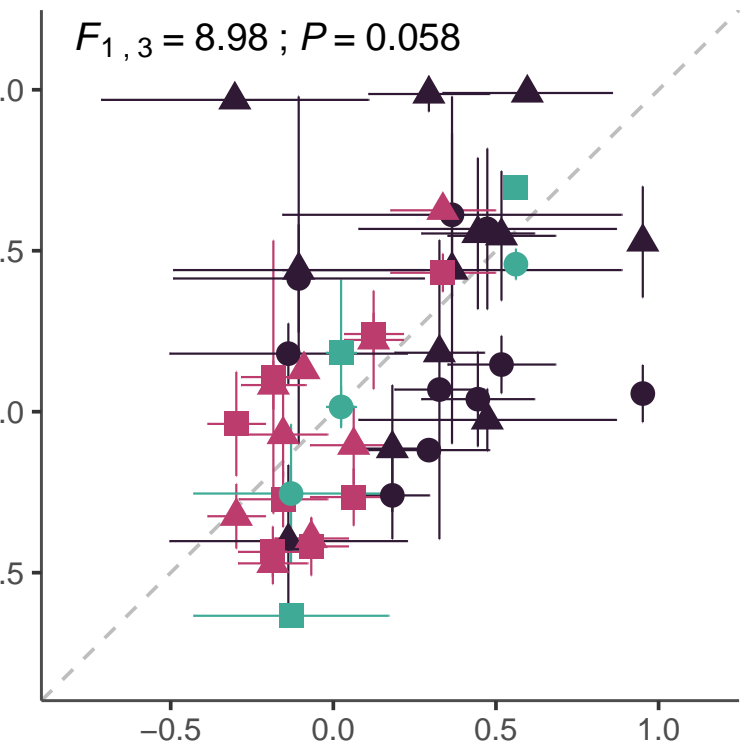

Offspring performance in sympatry
B

Generation G2/G3

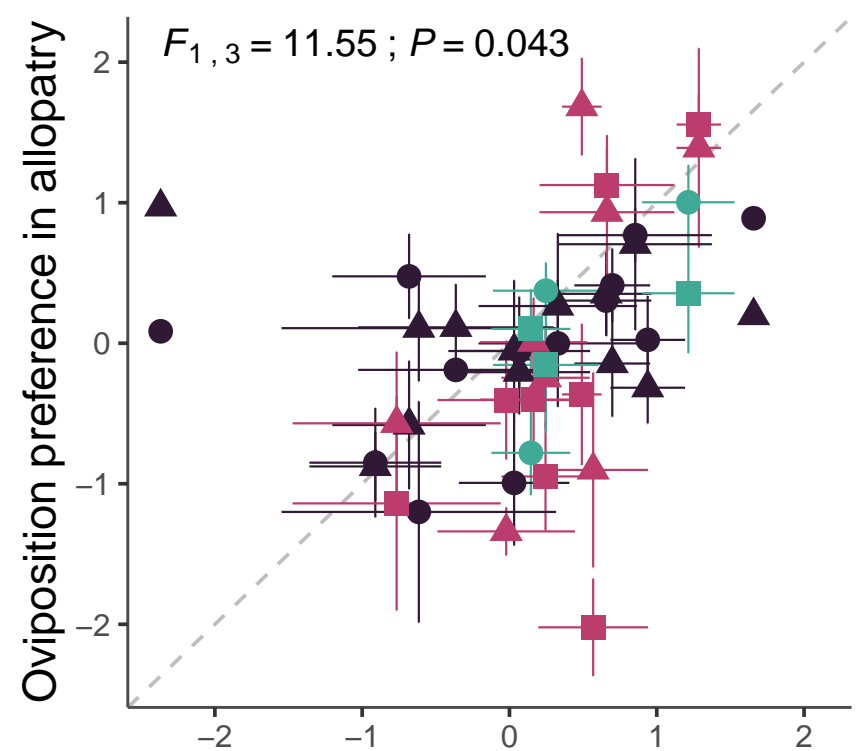

Oviposition preference in sympatry

D

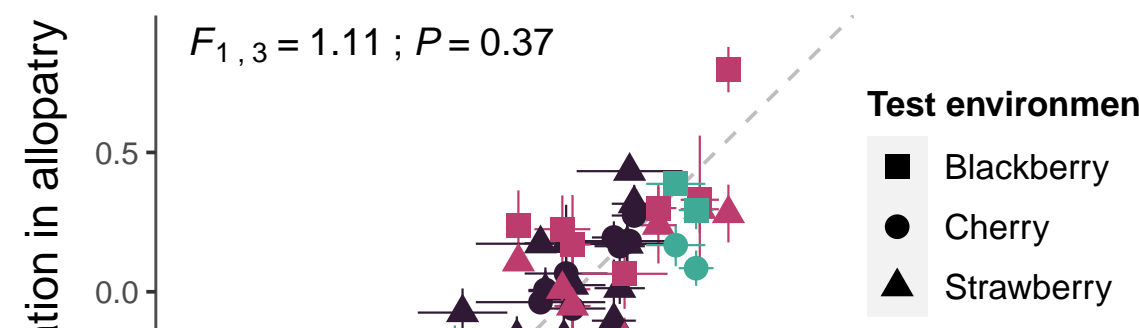

Fly population from:

Blackberry

Cherry

Strawberry

$\mathbf{F}$

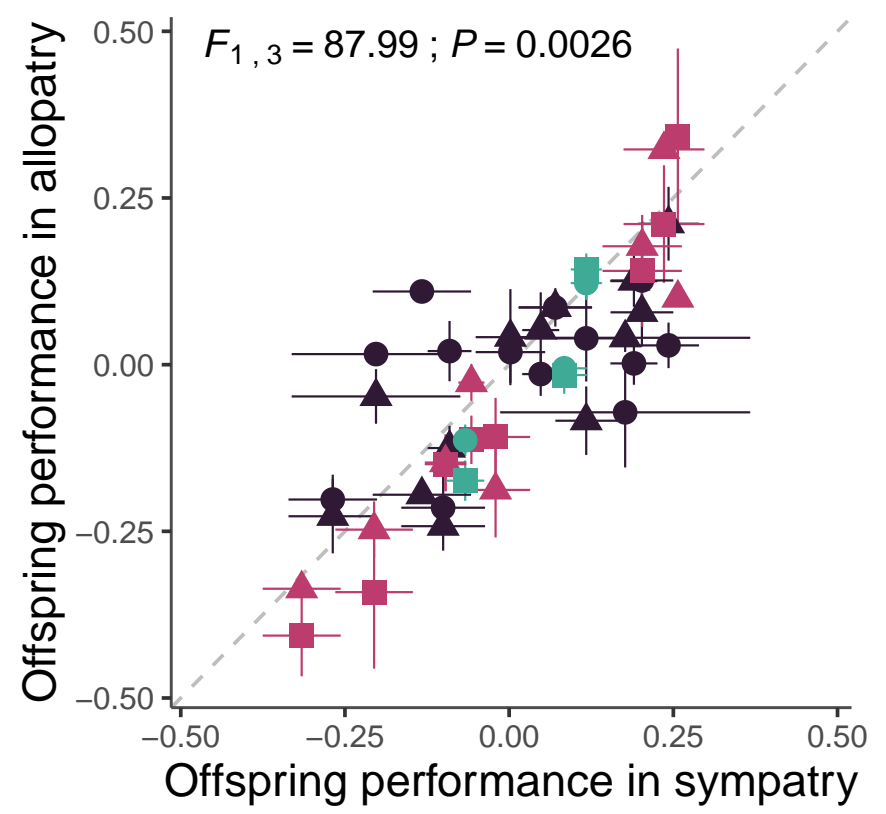


